\title{
KATO SMOOTHNESS AND FUNCTIONS OF PERTURBED SELF-ADJOINT OPERATORS
}

\author{
RUPERT L. FRANK AND ALEXANDER PUSHNITSKI
}

\begin{abstract}
We consider the difference $f\left(H_{1}\right)-f\left(H_{0}\right)$ for self-adjoint operators $H_{0}$ and $H_{1}$ acting in a Hilbert space. We establish a new class of estimates for the operator norm and the Schatten class norms of this difference. Our estimates utilise ideas of scattering theory and involve conditions on $H_{0}$ and $H_{1}$ in terms of the Kato smoothness. They allow for a much wider class of functions $f$ (including some unbounded ones) than previously available results do. As an important technical tool, we propose a new notion of Schatten class valued smoothness and develop a new framework for double operator integrals.
\end{abstract}

\section{INTRODUCTION}

1.1. Setting of the problem. Let $H_{0}$ and $H_{1}$ be self-adjoint operators in a Hilbert space $\mathcal{H}$, and let $f$ be a complex-valued function on the real line. In the framework of perturbation theory, the problem of estimating the difference

$$
D(f):=f\left(H_{1}\right)-f\left(H_{0}\right)
$$

either in the operator norm or in a Schatten class norm often arises. First, to set the scene, we display some known estimates in this context:

$$
\begin{aligned}
&\|D(f)\|_{p} \leq C(p)\|f\|_{\operatorname{Lip}(\mathbb{R})}\left\|H_{1}-H_{0}\right\|_{p}, \quad 1<p<\infty ; \\
&\|D(f)\|_{\mathcal{B}} \leq C\|f\|_{B_{\infty, 1}^{1}(\mathbb{R})}\left\|H_{1}-H_{0}\right\|_{\mathcal{B}}, \\
&\|D(f)\|_{1} \leq C\|f\|_{B_{\infty, 1}^{1}(\mathbb{R})}\left\|H_{1}-H_{0}\right\|_{1} .
\end{aligned}
$$

Here $\|\cdot\|_{p}$ is the norm in the standard Schatten class $\mathbf{S}_{p}$ and $\|\cdot\|_{\mathcal{B}}$ is the operator norm; $\operatorname{Lip}(\mathbb{R})$ is the Lipschitz class and $B_{\infty, 1}^{1}(\mathbb{R})$ is a Besov class. As usual, the case $p=2$ is very simple (and goes back at least to Birman and Solomyak in 1960s) and the important special cases $p=1$ and $p=\infty$ (i.e. (1.2) and (1.3)) are exceptional. The case $1<p<\infty$ is due to Potapov and Sukochev [15] and the cases $p=1$ and $p=\infty$ are due to Peller [13]. The estimate (1.1) is obviously sharp (Lip cannot be replaced by any larger class) and the estimates (1.2), (1.3) are very close to being sharp (the Besov class $B_{\infty, 1}^{1}(\mathbb{R})$ cannot be replaced by any larger Besov class).

In applications (we mainly have in mind the spectral theory of Schrödinger operators, see Section (1.5) one often has additional information on the perturbation $H_{1}-H_{0}$, which can be expressed in terms of conditions of the Kato smoothness

Date: 15 January 2019. 
type. In this paper, we propose a framework which allows one to systematically use these smoothness conditions in order to improve the estimates on $D(f)$, both in the operator norm and in the Schatten class norms.

1.2. Kato smoothness and the operator norm estimate. The notion of Kato smoothness was introduced by Kato in his seminal paper [10] (with further developments in [11]). In the same paper [10], it was used to prove the existence and completeness of wave operators. We will use this notion for a different purpose.

Let $H$ be a self-adjoint operator in a Hilbert space $\mathcal{H}$ and let $G$ be an operator acting from $\mathcal{H}$ to another Hilbert space $\mathcal{K}$. We will say that $G$ is Kato smooth with respect to $H$ (we will write $G \in \operatorname{Smooth}(H)$ ), if

$$
\|G\|_{\operatorname{Smooth}(H)}:=\sup _{\|\varphi\|_{L^{2}(\mathbb{R})}=1}\|G \varphi(H)\|_{\mathcal{B}}<\infty .
$$

This definition may look unfamiliar, but in fact we show in Section 2 that it coincides with the standard definition of Kato smoothness. We will see that the advantage of definition (1.4) is that it extends naturally to Schatten classes.

We start by stating, somewhat informally, our first (very simple) result; a more precise statement will be given in Section 6 .

Theorem 1.1. Let $H_{0}$ and $H_{1}$ be self-adjoint operators in $\mathcal{H}$ such that the perturbation $H_{1}-H_{0}$ factorises as

$$
H_{1}-H_{0}=G_{1}^{*} G_{0},
$$

with $G_{0} \in \operatorname{Smooth}\left(H_{0}\right)$ and $G_{1} \in \operatorname{Smooth}\left(H_{1}\right)$. Then for any $f \in \operatorname{BMO}(\mathbb{R})$, one has

$$
\|D(f)\|_{\mathcal{B}} \leq 2 \pi\|f\|_{\mathrm{BMO}(\mathbb{R})}\left\|G_{0}\right\|_{\operatorname{Smooth}\left(H_{0}\right)}\left\|G_{1}\right\|_{\operatorname{Smooth}\left(H_{1}\right)} .
$$

Here $\mathrm{BMO}(\mathbb{R})$ is the class of functions with bounded mean oscillation; we recall the description of this class in Section 5 and fix a suitable norm on it (there are many equivalent norms on $\mathrm{BMO}(\mathbb{R})$, but by choosing a specific norm, we make the constant in front of the right hand side of (1.5) equal to $2 \pi$ ).

Observe that functions in $\mathrm{BMO}(\mathbb{R})$ include some unbounded ones, such as $f(\lambda)=\log |\lambda|$. This is in sharp contrast with the estimate (1.2), where $f$ has to be bounded, continuous and everywhere differentiable (see e.g. [1] for the differentiability statement).

1.3. $\mathbf{S}_{p}$-valued smoothness and Schatten norm estimates. For $0<p<\infty$, let $\mathbf{S}_{p}$ be the standard Schatten class with the (quasi-)norm $\|\cdot\|_{p}$ (see Section 1.7 for the definition). Generalising (1.4), we will say that $G \in \operatorname{Smooth}_{p}(H)$ if

$$
\|G\|_{\operatorname{Smooth}_{p}(H)}:=\sup _{\|\varphi\|_{L^{2}(\mathbb{R})}=1}\|G \varphi(H)\|_{p}<\infty .
$$

Our main result is the following Schatten class estimate (it will be restated more precisely as Theorem 6.5 in Section 6). 
Theorem 1.2. Let $p, q, r$ be finite positive numbers such that $\frac{1}{p}=\frac{1}{q}+\frac{1}{r}$. Let $H_{1}$ and $H_{0}$ be self-adjoint operators in $\mathcal{H}$ such that $H_{1}-H_{0}={ }_{G}^{*} G_{0}$ with $G_{0} \in$ $\operatorname{Smooth}_{q}\left(H_{0}\right), G_{1} \in \operatorname{Smooth}_{r}\left(H_{1}\right)$. Then for all $f \in B_{p, p}^{1 / p}(\mathbb{R}) \cap \operatorname{BMO}(\mathbb{R})$, one has

$$
\|D(f)\|_{p} \leq C(p)\|f\|_{B_{p, p}^{1 / p}(\mathbb{R})}\left\|G_{0}\right\|_{\operatorname{Smooth}_{q}\left(H_{0}\right)}\left\|G_{1}\right\|_{\operatorname{Smooth}_{r}\left(H_{1}\right)} .
$$

This extends to $q=\infty$ (resp. to $r=\infty$ ), if one replaces $\operatorname{Smooth}_{q}\left(H_{0}\right)$ (resp. $\left.\operatorname{Smooth}_{r}\left(H_{1}\right)\right)$ by $\operatorname{Smooth}\left(H_{0}\right)$ (resp. by $\left.\operatorname{Smooth}\left(H_{1}\right)\right)$.

We recall the definition of the Besov class $B_{p, p}^{1 / p}(\mathbb{R})$ in Section 5 . The constant $C(p)$ in (1.6) depends only on the choice of the functional $\|\cdot\|_{B_{p, p}^{1 / p}(\mathbb{R})}$ in this class. We say "the functional" rather than "the norm", because technically $\|\cdot\|_{B_{p, p}^{1 / p}(\mathbb{R})}$ is a semi-(quasi)norm; semi because it vanishes on all polynomials and quasi because it satisfies the triangle inequality of the form

$$
\begin{array}{ll}
\|f+g\|_{B_{p, p}^{1 / p}(\mathbb{R})} \leq\|f\|_{B_{p, p}^{1 / p}(\mathbb{R})}+\|g\|_{B_{p, p}^{1 / p}(\mathbb{R})}, & p \geq 1, \\
\|f+g\|_{B_{p, p}^{1 / p}(\mathbb{R})}^{p} \leq\|f\|_{B_{p, p}^{1 / p}(\mathbb{R})}^{p}+\|g\|_{B_{p, p}^{1 / p}(\mathbb{R})}^{p}, \quad 0<p<1 .
\end{array}
$$

Requiring that $f \in \mathrm{BMO}(\mathbb{R})$ reduces the arbitrary polynomial in the definition of $f$ to an arbitrary additive constant. Observe that for $f=$ const, we have $D(f)=0$.

To illustrate the type of local singularities allowed for functions $f \in B_{p, p}^{1 / p}(\mathbb{R})$, consider the following example. Let $\chi_{0} \in C_{0}^{\infty}(\mathbb{R})$ be a function which equals 1 in a neighbourhood of the origin and vanishes outside the interval $(-c, c)$ with some $0<c<1$. Fix $\alpha \in \mathbb{R}, a_{+}, a_{-} \in \mathbb{C}$, and consider the function

$$
F_{\alpha}(x)= \begin{cases}a_{+} \chi_{0}(x)|\log | x||^{-\alpha}, & x>0, \\ a_{-} \chi_{0}(x)|\log | x||^{-\alpha}, & x<0 .\end{cases}
$$

Proposition 1.3. Let $F_{\alpha}$ be as defined above and let $0<p<\infty$.

(i) If $a_{+} \neq a_{-}$, then $f \in B_{p, p}^{1 / p}(\mathbb{R})$ if and only if $\alpha>1 / p$.

(ii) If $a_{+}=a_{-} \neq 0$, then $f \in B_{p, p}^{1 / p}(\mathbb{R})$ if and only if $\alpha+1>1 / p$.

In essence, this is an elementary computation using the definition of $B_{p, p}^{1 / p}(\mathbb{R})$; we sketch the proof in the Appendix.

Again, we see that for $p>1$, the functions $F_{\alpha} \in B_{p, p}^{1 / p}(\mathbb{R})$ may be unbounded. It is also clear that, in contrast with (1.1), $F_{\alpha}$ is never in Lip, apart from the trivial cases $a_{+}=a_{-}=0$ or $\alpha=0$.

We note briefly that Theorems 1.1 and 1.2 are sharp in the sense that the corresponding estimates are saturated for certain operators $H_{0}$ and $H_{1}$; see Theorem 7.1 below.

1.4. Key ideas of the proof. For a function $f: \mathbb{R} \rightarrow \mathbb{C}$, we denote by $\check{f}$ the divided difference

$$
\check{f}(x, y):=\frac{f(x)-f(y)}{x-y}, \quad x, y \in \mathbb{R} .
$$


The Birman-Solomyak formula, which goes back to [2] (see also [3] for a modern exposition), represents the difference $D(f)$ as the double operator integral (DOI):

$$
D(f)=\int_{\mathbb{R}} \int_{\mathbb{R}} \check{f}(x, y) d E_{H_{1}}(x)\left(H_{1}-H_{0}\right) d E_{H_{0}}(y),
$$

where $E_{H_{0}}$ (resp. $E_{H_{1}}$ ) is the projection-valued spectral measure of $H_{0}$ (resp. of $H_{1}$ ). The standard approach (which again goes back to Birman and Solomyak) to the problem of estimating the norm of $D(f)$ is to represent the map $f \mapsto D(f)$ as a composition of two maps,

$$
f \mapsto \check{f} \mapsto \int_{\mathbb{R}} \int_{\mathbb{R}} \check{f}(x, y) d E_{H_{1}}(x)\left(H_{1}-H_{0}\right) d E_{H_{0}}(y) .
$$

To explain this further, let us first recall the strategy of the proof of the estimate (1.2). One proves (see [13]) separately the estimates

$$
\begin{aligned}
&\left\|\int_{\mathbb{R}} \int_{\mathbb{R}} \check{f}(x, y) d E_{H_{1}}(x)\left(H_{1}-H_{0}\right) d E_{H_{0}}(y)\right\| \leq C\|\check{f}\|_{*}\left\|H_{1}-H_{0}\right\|_{\mathcal{B}}, \\
&\|\check{f}\|_{*} \leq C\|f\|_{B_{\infty, 1}^{1}(\mathbb{R})},
\end{aligned}
$$

where $\|\cdot\|_{*}$ is a certain norm on the set of integral kernels (functions of two variables). Putting them together and using the Birman-Solomyak formula, this yields (1.2).

We use the composition (1.9) as well, but the underlying estimates are different. Essentially, we develop an alternative version of the theory of DOI as follows. We fix $H_{0}, H_{1}$ and $G_{0} \in \operatorname{Smooth}\left(H_{0}\right), G_{1} \in \operatorname{Smooth}\left(H_{1}\right)$ and consider the map

$$
a \mapsto \int_{\mathbb{R}} \int_{\mathbb{R}} a(x, y) d E_{H_{1}}(x) G_{1}^{*} G_{0} d E_{H_{0}}(y) ;
$$

here $a$ is an arbitrary bounded operator in $L^{2}(\mathbb{R})$ with the integral kernel $a(x, y)$. We prove the estimates

$$
\begin{aligned}
\left\|\int_{\mathbb{R}} \int_{\mathbb{R}} a(x, y) d E_{H_{1}}(x) G_{1}^{*} G_{0} d E_{H_{0}}(y)\right\| & \leq\|a\|_{\mathcal{B}}\left\|G_{0}\right\|_{\operatorname{Smooth}\left(H_{0}\right)}\left\|G_{1}\right\|_{\operatorname{Smooth}\left(H_{1}\right)}, \\
\|\check{f}\|_{\mathcal{B}} & \leq 2 \pi\|f\|_{\mathrm{BMO}(\mathbb{R})} .
\end{aligned}
$$

These estimates, together with the Birman-Solomyak formula, yield the proof of Theorem 1.1. Theorem 1.2 is obtained from the Schatten class versions of (1.10) and (1.11).

We note that while (1.10) (and its Schatten class version) is new, the estimate (1.11) is essentially well known. In fact, the operator with the integral kernel $\breve{f}(x, y)$ is a Hankel operator in disguise; this is well known in the Hankel operator community, and (1.11) easily follows from there. 
1.5. Some applications. Here we briefly mention some applications of Theorems 1.1 and 1.2, these are developed in detail in the forthcoming publication [6]. Let

$$
H_{0}=-\Delta, \quad H_{1}=-\Delta+V \quad \text { in } L^{2}\left(\mathbb{R}^{d}\right), d \geq 1,
$$

where the real-valued potential $V$ satisfies the bound

$$
|V(x)| \leq C(1+|x|)^{-\rho}, \quad \rho>1 .
$$

Under these assumptions, the absolutely continuous spectrum of both $H_{0}$ and $H_{1}$ coincides with $[0, \infty)$. In applications to mathematical physics (see e.g. [5]), one is often interested in functions $f$ having a cusp-type singularity on the absolutely continuous spectrum and smooth elsewhere. It is also easy to reduce the question to functions $f$ compactly supported on $(0, \infty)$.

Theorem 1.4. 6]

(i) Assume $\rho>1$. Then any $f \in \operatorname{BMO}(\mathbb{R})$ with compact support in $(0, \infty)$, we have $D(f) \in \mathcal{B}$.

(ii) Assume $1<\rho \leq d$. Then for any $p>\frac{d-1}{\rho-1}$ and for any $f \in B_{p, p}^{1 / p}(\mathbb{R})$ with compact support in $(0, \infty)$, we have $D(f) \in \mathbf{S}_{p}$.

(iii) Assume $\rho>d$. Then for any $p>d / \rho$ and for any $f \in B_{p, p}^{1 / p}(\mathbb{R})$ with compact support in $(0, \infty)$, we have $D(f) \in \mathbf{S}_{p}$.

In the $p=1$ case, this is the result of our previous publication [5].

In the proof of Theorem 1.4, the concept of local $\mathbf{S}_{p}$-valued smoothness is important; in other words, one needs inclusions of the type $G E_{H_{0}}(\Delta) \in \operatorname{Smooth}_{p}\left(H_{0}\right)$, where $\Delta \subset(0, \infty)$. We develop some tools for this in Section 7.3 .

1.6. The structure of the paper. In Section 2 we discuss the classical Kato smoothness and in Section 3 we introduce and study the $\mathbf{S}_{p}$-valued smoothness. In Section 4 we develop our version of the theory of DOI and prove the estimate (1.10) and its Schatten class version. The key idea of the proof is a certain factorisation of the DOI and a subsequent use of interpolation on each factor. In Section 5 we derive the estimate (1.11) (and its Schatten class version) from the known estimates for Hankel operators. In Section 6 we put all the components together and prove Theorems 1.1 and 1.2. Section 7 contains some additional information. First we present an example which illustrates the sharpness of our main results. Then we consider some extensions: to "quasicommutators"

$$
f\left(H_{1}\right) J-J f\left(H_{0}\right)
$$

and to operators of the form

$$
\varphi_{1}\left(H_{1}\right)^{*}\left(f\left(H_{1}\right)-f\left(H_{0}\right)\right) \varphi_{0}\left(H_{0}\right) .
$$


The latter operator is important in applications, which we develop in a separate paper [6]. In Appendix, we sketch the proof of Proposition 1.3 and of another technical statement of a similar nature.

1.7. Notation. Throughout the paper, $\mathcal{H}$ and $\mathcal{K}$ are complex separable Hilbert spaces. If $H$ is a self-adjoint operator in $\mathcal{H}$, then $E_{H}(\Lambda)=\mathbb{1}_{\Lambda}(H)$ is the spectral projection of $H$ associated to the set $\Lambda \subset \mathbb{R}$. Here and in what follows $\mathbb{1}_{\Lambda}$ is the characteristic function of the set $\Lambda$. We denote by $\mathcal{H}^{(\mathrm{ac})}(H)\left(\right.$ resp. $\left.\mathcal{H}^{(\mathrm{sing})}(H)\right)$ the absolutely continuous (resp. singular) subspace of $H$, and $H^{(\mathrm{ac})}=\left.H\right|_{\mathcal{H}^{(\mathrm{ac})}(H)}$.

We will often deal with weakly convergent sequences of bounded operators in a Hilbert space, i.e. $\left(A_{n} x, y\right) \rightarrow(A x, y)$ for all elements $x, y$ in the Hilbert space. Recall that this is equivalent to $\operatorname{Tr}\left(A_{n} B\right) \rightarrow \operatorname{Tr}(A B)$ for all trace class operators $B$. Thus, for the sake of uniformity with other types of convergences in function spaces, we shall call this $*$-weak convergence in the set of bounded operators.

The set of bounded operators acting from $\mathcal{H}$ to $\mathcal{K}$ is denoted by $\mathcal{B}(\mathcal{H}, \mathcal{K})$, and the corresponding norm is denoted by $\|\cdot\|_{\mathcal{B}}$. We use the class of compact operators $\mathbf{S}_{\infty}(\mathcal{H}, \mathcal{K})$ acting from $\mathcal{H}$ to $\mathcal{K}$ and, for $0<p<\infty$, the Schatten class $\mathbf{S}_{p}(\mathcal{H}, \mathcal{K}) \subset$ $\mathbf{S}_{\infty}(\mathcal{H}, \mathcal{K})$, defined by

$$
\|A\|_{p}^{p}=\sum_{n=1}^{\infty} s_{n}(A)^{p}<\infty,
$$

where $\left\{s_{n}(A)\right\}_{n=1}^{\infty}$ is the sequence of singular values of $A$, enumerated with multiplicities taken into account. Observe that $\|\cdot\|_{p}$ is a norm for $p \geq 1$, and a quasinorm for $0<p<1$; the triangle inequality fails in the latter case. However, for $0<p<1$ there is a useful substitute for the triangle inequality due to Rotfeld [19] (see also [12])

$$
\|A+B\|_{p}^{p} \leq\|A\|_{p}^{p}+\|B\|_{p}^{p}, \quad 0<p<1 .
$$

We frequently use the "Hölder inequality for $\mathbf{S}_{p}$ classes"

$$
\|A B\|_{p} \leq\|A\|_{q}\|B\|_{r}, \quad \frac{1}{p}=\frac{1}{q}+\frac{1}{r} .
$$

Acknowledgements. Partial support by U.S. National Science Foundation DMS-1363432 (R.L.F.) is acknowledged. We are grateful to Barry Simon for discussions related to the proof of Theorem 3.3. A.P. is grateful to Caltech for hospitality.

\section{KATO SMOOTHNESS}

Let $H$ be a self-adjoint operator in $\mathcal{H}$, and let $G: \mathcal{H} \rightarrow \mathcal{K}$ be an $H$-bounded operator; that is, Dom $H \subset \operatorname{Dom} G$ and the operator $G R(z)$ is bounded for all $\operatorname{Im} z \neq 0$; here and in what follows we denote $R(z)=(H-z)^{-1}$.

Note that the operator $G$ is not assumed to be closed or closable; in fact, in one of our examples $G$ will not admit closure. So the stand-alone adjoint $G^{*}$ is not necessarily well defined, but products of the type $(G R(z))^{*}$ are. 
2.1. Kato smoothness. We recall (see e.g. [20, Section 4.3]) that for an $H$ bounded operator $G$, the following conditions are equivalent:

$$
\begin{gathered}
c_{1}:=(2 \pi)^{-2} \sup _{\varepsilon>0,\|u\|=1} \int_{\mathbb{R}}\left(\|G R(x+i \varepsilon) u\|_{\mathcal{K}}^{2}+\|G R(x-i \varepsilon) u\|_{\mathcal{K}}^{2}\right) d x<\infty \\
c_{2}:=\sup _{\varepsilon>0,\|u\|=1} \frac{\varepsilon^{2}}{\pi^{2}} \int_{\mathbb{R}}\left(\|G R(x+i \varepsilon) R(x-i \varepsilon) u\|_{\mathcal{K}}^{2} d x<\infty\right. \\
c_{3}:=\sup _{(a, b) \subset \mathbb{R}} \frac{\left\|G E_{H}(a, b)\right\|_{\mathcal{B}}^{2}}{|b-a|}<\infty .
\end{gathered}
$$

If these conditions hold true, then

$$
c_{1}=c_{2}=c_{3} .
$$

In this case, the operator $G$ is called $H$-smooth, and we will write $G \in \operatorname{Smooth}(H)$. We will denote

$$
\|G\|_{\operatorname{Smooth}(H)}:=\sqrt{c_{1}}=\sqrt{c_{2}}=\sqrt{c_{3}} .
$$

We recall that for $G \in \operatorname{Smooth}(H)$, one has $\left.G\right|_{\mathcal{H}^{(\text {sing })(H)}}=0$; here $\mathcal{H}^{(\text {sing })}(H)$ is the singular subspace of $H$.

As mentioned in the Introduction, we will need a slightly non-standard equivalent definition of smoothness, given by the following theorem.

Theorem 2.1. $G \in \operatorname{Smooth}(H)$ if and only if

$$
\|G \varphi(H)\|_{\mathcal{B}} \leq C\|\varphi\|_{L^{2}}, \quad \forall \varphi \in L^{2}(\mathbb{R}) .
$$

Further, in this case the norm $\|G\|_{\operatorname{Smooth}(H)}$ coincides with the optimal constant in (2.4):

$$
\|G\|_{\operatorname{Smooth}(H)}=\sup _{\|\varphi\|_{L^{2}}=1}\|G \varphi(H)\| .
$$

Before proving this theorem, we need to address a minor technical issue: since the operator $\varphi(H)$ is in general unbounded, the definition of $G \varphi(H)$ must be made more precise. We define $G \varphi(H)$ to be zero on $\mathcal{H}^{(\operatorname{sing})}(H)$. Next, we will denote by $L_{\text {comp }}^{\infty}(H)$ the set of all elements $u \in \mathcal{H}^{(\text {ac) }}(H)$ for which the function

$$
\frac{d\left(E_{H}(-\infty, \lambda) u, u\right)}{d \lambda}, \quad \lambda \in \mathbb{R}
$$

is compactly supported and uniformly bounded on $\mathbb{R}$. It is not difficult to show that $L_{\text {comp }}^{\infty}(H)$ is dense in $\mathcal{H}^{(\text {ac) }}(H)$. It is also easy to see that for $u \in L_{\text {comp }}^{\infty}(H)$, the element $\varphi(H) u$ is defined for $\varphi \in L_{\mathrm{loc}}^{2}(\mathbb{R})$ and we have $\varphi(H) u \in \operatorname{Dom}(H)$. Thus, $G \varphi(H) u$ is well defined for $u \in L_{\text {comp }}^{\infty}(H)$. Theorem 2.1 says that this definition can be extended to all $u \in \mathcal{H}^{(\mathrm{ac})}(H)$ with the norm bound (2.4) if and only if $G \in \operatorname{Smooth}(H)$. 
Proof of Theorem 2.1. Assume that $G \in \operatorname{Smooth}(H)$; let us prove (2.4). It suffices to consider the dense set of functions $\varphi$ of the form

$$
\varphi=\sum_{k} \varphi_{k} \mathbb{1}_{\Lambda_{k}}
$$

where the sum is finite, $\Lambda_{k}$ are disjoint intervals in $\mathbb{R}$ and $\varphi_{k} \in \mathbb{C}$. Then, by (2.3),

$$
\begin{aligned}
\|G \varphi(H)\|_{\mathcal{B}}^{2}=\left\|G \varphi(H)(G \varphi(H))^{*}\right\|_{\mathcal{B}} \leq \sum_{k}\left|\varphi_{k}\right|^{2}\left\|G E_{H}\left(\Lambda_{k}\right)\left(G E_{H}\left(\Lambda_{k}\right)\right)^{*}\right\|_{\mathcal{B}} \\
\leq\|G\|_{\operatorname{Smooth}(H)}^{2} \sum_{k}\left|\Lambda_{k}\left\|\left.\varphi_{k}\right|^{2}=\right\| G\left\|_{\operatorname{Smooth}(H)}^{2}\right\| \varphi \|_{L^{2}}^{2},\right.
\end{aligned}
$$

and so we obtain (2.4) with $C=\|G\|_{\operatorname{Smooth}(H)}$. The converse follows by taking $\varphi=\mathbb{1}_{(a, b)}$ and by comparing with (2.3) .

An important ingredient of our construction is

Theorem 2.2. Let $G \in \operatorname{Smooth}(H)$ and let $\left\{\psi_{n}\right\}_{n=1}^{\infty}$ be an orthonormal sequence in $L^{2}(\mathbb{R})$. Then for any $u \in \mathcal{H}$ :

$$
\sum_{n=1}^{\infty}\left\|G \psi_{n}(H) u\right\|_{\mathcal{K}}^{2} \leq\|G\|_{\operatorname{Smooth}(H)}^{2}\|u\|_{\mathcal{H}}^{2} .
$$

Proof. Denote by $P_{\varepsilon}$ the Poisson kernel,

$$
P_{\varepsilon}(x)=\frac{\varepsilon}{\pi\left(x^{2}+\varepsilon^{2}\right)}, \quad x \in \mathbb{R}, \quad \varepsilon>0 .
$$

For $\varepsilon>0$, let $F_{\varepsilon}(x)=G P_{\varepsilon}(H-x) u, x \in \mathbb{R}$. Then by $(2.2), F_{\varepsilon} \in L^{2}(\mathbb{R} ; \mathcal{K})$ with the norm estimate

$$
\int_{\mathbb{R}}\left\|F_{\varepsilon}(x)\right\|_{\mathcal{K}}^{2} d x \leq\|G\|_{\operatorname{Smooth}(H)}^{2}\|u\|_{\mathcal{H}}^{2} .
$$

Let $N \in \mathbb{N}$ and let $v_{1}, \ldots, v_{N} \in \mathcal{K}$ be any set of elements with $\left\|v_{n}\right\|=1$ for each $n$. Then the set $\left\{\overline{\psi_{n}(x)} v_{n}\right\}_{n=1}^{N}$ is orthonormal in the space $L^{2}(\mathbb{R} ; \mathcal{K})$, and therefore, by the Cauchy-Schwarz in the same space,

$$
\sum_{n=1}^{N}\left|\left(v_{n}, \int_{\mathbb{R}} \psi_{n}(x) F_{\varepsilon}(x) d x\right)_{\mathcal{K}}\right|^{2}=\sum_{n=1}^{N}\left|\left(\overline{\psi_{n}} v_{n}, F_{\varepsilon}\right)_{L^{2}(\mathbb{R} ; \mathcal{K})}\right|^{2} \leq \int_{\mathbb{R}}\left\|F_{\varepsilon}(x)\right\|_{\mathcal{K}}^{2} d x .
$$

Choosing

$$
v_{n}=c_{n} \int_{\mathbb{R}} \psi_{n}(x) F_{\varepsilon}(x) d x
$$

with a suitable normalisation constant $c_{n}$, from here we obtain

$$
\sum_{n=1}^{N}\left\|\int_{\mathbb{R}} \psi_{n}(x) F_{\varepsilon}(x) d x\right\|_{\mathcal{K}}^{2} \leq \int_{\mathbb{R}}\left\|F_{\varepsilon}(x)\right\|_{\mathcal{K}}^{2} d x \leq\|G\|_{\operatorname{Smooth}(H)}^{2}\|u\|_{\mathcal{H}}^{2}
$$


for every $N \in \mathbb{N}$. Next, for every $n \geq 1$, we have

$$
\int_{\mathbb{R}} \psi_{n}(x) F_{\varepsilon}(x) d x=\int_{\mathbb{R}} \psi_{n}(x) G P_{\varepsilon}(H-x) u d x=G \psi_{n}^{(\varepsilon)}(H) u
$$

where

$$
\psi_{n}^{(\varepsilon)}(x)=\int_{\mathbb{R}} \psi_{n}(t) P_{\varepsilon}(x-t) d t
$$

Thus, (2.7) can be written as

$$
\sum_{n=1}^{N}\left\|G \psi_{n}^{(\varepsilon)}(H) u\right\|_{\mathcal{K}}^{2} \leq\|G\|_{\operatorname{Smooth}(H)}^{2}\|u\|_{\mathcal{H}}^{2} .
$$

Further, by the properties of the Poisson kernel, $\left\|\psi_{n}^{(\varepsilon)}-\psi_{n}\right\|_{L^{2}} \rightarrow 0$ as $\varepsilon \rightarrow 0$ for all $n$, and therefore, by Theorem 2.1,

$$
\left\|G \psi_{n}^{(\varepsilon)}(H) u-G \psi_{n}(H) u\right\|_{\mathcal{K}} \rightarrow 0, \quad \varepsilon \rightarrow 0
$$

for all $n$. It follows that for any $N$,

$$
\sum_{n=1}^{N}\left\|G \psi_{n}(H) u\right\|_{\mathcal{K}}^{2} \leq\|G\|_{\operatorname{Smooth}(H)}^{2}\|u\|_{\mathcal{H}}^{2} .
$$

Since $N$ is arbitrary, we obtain (2.5).

2.2. The class $\operatorname{Smooth}_{\infty}(H)$. We will write $G \in \operatorname{Smooth}_{\infty}(H)$, if $G \in \operatorname{Smooth}(H)$ and if

$$
G E_{H}(-R, R) \in \mathbf{S}_{\infty} \quad \forall R>0 .
$$

Lemma 2.3. Let $G \in \operatorname{Smooth}_{\infty}(H)$; then $G \varphi(H)$ is compact for any $\varphi \in L^{2}(\mathbb{R})$.

Proof. Since $G E_{H}(-R, R)$ is compact for any $R>0$, the operator $G \varphi\left(H_{0}\right)$ is compact for $\varphi \in L_{\text {comp }}^{\infty}(\mathbb{R})$. Since $L_{\text {comp }}^{\infty}$ is dense in $L^{2}$, the bound (2.4) implies that $G \varphi\left(H_{0}\right)$ is compact for all $\varphi \in L^{2}$, as claimed.

2.3. Smoothness with respect to the multiplication operator. It will be important for us to have a description of the class $\operatorname{Smooth}(\mathcal{M})$, where $\mathcal{M}$ is the operator of multiplication by the independent variable in a vector-valued $L^{2}$-space. Such description was given by Kato in [11. Let $\mathfrak{h}$ be an auxiliary Hilbert space (which may be finite or infinite dimensional), and let $\mathcal{H}=L^{2}(\mathbb{R} ; \mathfrak{h})$ be the $L^{2}$ space of $\mathfrak{h}$-valued functions. The operator $\mathcal{M}$ in $\mathcal{H}$ is defined as

$$
\begin{gathered}
(\mathcal{M} f)(x)=x f(x), \quad f \in \operatorname{Dom} \mathcal{M}, \\
\operatorname{Dom} \mathcal{M}=\left\{f \in L^{2}(\mathbb{R} ; \mathfrak{h}): \int_{\mathbb{R}}\|f(x)\|_{\mathfrak{h}}^{2}\left(x^{2}+1\right) d x<\infty\right\} .
\end{gathered}
$$


Theorem 2.4. [11] Let $\mathcal{M}$ be as above and let $G: \mathcal{H} \rightarrow \mathcal{K}$ be an $\mathcal{M}$-bounded operator. Then $G \in \operatorname{Smooth}(\mathcal{M})$ if and only if $G$ can be represented as

$$
G f=\int_{\mathbb{R}} g(x) f(x) d x, \quad \forall f \in \operatorname{Dom} \mathcal{M},
$$

with some $g \in L^{\infty}(\mathbb{R} ; \mathcal{B}(\mathfrak{h}, \mathcal{K}))$. Moreover, in this case we have the equality of the norms

$$
\|G\|_{\operatorname{Smooth}(\mathcal{M})}=\|g\|_{L^{\infty}(\mathbb{R} ; \mathcal{B}(\mathfrak{h}, \mathcal{K}))} .
$$

This theorem plays a crucial role in our construction; see Theorem 3.3 and Lemma 3.6 below. For this reason and for the sake of completeness we give a proof, which is essentially a rewording of Kato's proof in [11].

Proof. Let $g \in L^{\infty}(\mathbb{R} ; \mathcal{B}(\mathfrak{h}, \mathcal{K}))$ and let $G$ be defined according to (2.10). Then it is clear that for every finite interval $\Lambda$ the operator $G E_{\mathcal{M}}(\Lambda)$ is bounded and

$$
G E_{\mathcal{M}}(\Lambda)\left(G E_{\mathcal{M}}(\Lambda)\right)^{*}=\int_{\Lambda} g(x) g(x)^{*} d x
$$

It follows that

$$
\left\|G E_{\mathcal{M}}(\Lambda)\right\|_{\mathcal{B}(\mathcal{H}, \mathcal{K})}^{2}=\left\|\int_{\Lambda} g(x) g(x)^{*} d x\right\|_{\mathcal{B}(\mathcal{K})} \leq|\Lambda| \sup _{x}\|g(x)\|_{\mathcal{B}(\mathfrak{h}, \mathcal{K})}^{2},
$$

and so, by (2.3),$G \in \operatorname{Smooth}(\mathcal{M})$ and

$$
\|G\|_{\operatorname{Smooth}(\mathcal{M})} \leq\|g\|_{L^{\infty}(\mathbb{R} ; \mathcal{B}(\mathfrak{h}, \mathcal{K}))} .
$$

Conversely, let $G \in \operatorname{Smooth}(\mathcal{M})$. First we need an auxiliary estimate. Observe that $\operatorname{Dom} \mathcal{M} \subset L^{1}(\mathbb{R} ; \mathfrak{h})$. Write every $f \in \operatorname{Dom} \mathcal{M}$ as $f=f_{1}(\mathcal{M}) f_{2}$, where

$$
f_{1}(x)=\|f(x)\|_{\mathfrak{h}}^{1 / 2} \quad \text { and } \quad f_{2}(x)=\|f(x)\|_{\mathfrak{h}}^{-1 / 2} f(x) \quad \text { for a.e. } x \in \mathbb{R} .
$$

Then $f_{1} \in L^{2}(\mathbb{R}), f_{2} \in L^{2}(\mathbb{R} ; \mathfrak{h})$ and

$$
\left\|f_{1}\right\|_{L^{2}(\mathbb{R})}^{2}=\left\|f_{2}\right\|_{L^{2}(\mathbb{R} ; \mathfrak{h})}^{2}=\|f\|_{L^{1}(\mathbb{R} ; \mathfrak{h})} .
$$

By Theorem 2.1, we obtain

$$
\begin{aligned}
\|G f\|=\left\|G f_{1}(\mathcal{M}) f_{2}\right\| \leq\|G\|_{\operatorname{Smooth}(\mathcal{M})}\left\|f_{1}\right\|_{L^{2}(\mathbb{R})}\left\|f_{2}\right\|_{L^{2}(\mathbb{R} ; \mathfrak{h})} \\
=\|G\|_{\operatorname{Smooth}(\mathcal{M})}\|f\|_{L^{1}(\mathbb{R} ; \mathfrak{h})} .
\end{aligned}
$$

Now let us establish the existence of $g \in L^{\infty}(\mathbb{R} ; \mathcal{B}(\mathfrak{h}, \mathcal{K}))$ that satisfies (2.10). In order to define the function $g(x)$, it is easier to start with the adjoint $g(x)^{*}$. Let $\psi \in \mathcal{K}$; by (2.14), we have

$$
|(G f, \psi)| \leq\|G\|_{\operatorname{Smooth}(\mathcal{M})}\|\psi\|_{\mathcal{K}}\|f\|_{L^{1}(\mathbb{R} ; \mathfrak{h})} .
$$

It follows that the linear functional $f \mapsto(G f, \psi)$ is bounded on $L^{1}(\mathbb{R} ; \mathfrak{h})$ and therefore (see e.g. [9, Corollary 1.3.22]) it can be represented as

$$
(G f, \psi)=\int_{\mathbb{R}}\left(f(x), g_{\psi}(x)\right)_{\mathfrak{h}} d x
$$


with some $g_{\psi} \in L^{\infty}(\mathbb{R} ; \mathfrak{h})$ satisfying

$$
\left\|g_{\psi}\right\|_{L^{\infty}(\mathbb{R} ; \mathfrak{h})} \leq\|G\|_{\operatorname{Smooth}(\mathcal{M})}\|\psi\|_{\mathcal{K}}
$$

By the uniqueness of this representation, $g_{\psi}$ depends linearly on $\psi$. Now for $x \in \mathbb{R}$, let us define the operator $g(x)^{*}: \mathcal{K} \rightarrow \mathfrak{h}$ by

$$
g(x)^{*} \psi:=g_{\psi}(x)
$$

(to be precise, this should be done on a suitable countable dense set of $\psi$ and a suitable set of $x$ of full measure - we omit these details). By (2.16), we have

$$
\operatorname{ess} \sup _{x}\left\|g^{*}(x)\right\|_{\mathcal{B}(\mathcal{K}, \mathfrak{h})} \leq\|G\|_{\operatorname{Smooth}(\mathcal{M})} .
$$

Now we can define $g(x): \mathfrak{h} \rightarrow \mathcal{K}$ as the adjoint of $g(x)^{*}$. From (2.15) we obtain that

$$
\int_{\mathbb{R}}(g(x) f(x), \psi)_{\mathcal{K}} d x=(G f, \psi)_{\mathcal{K}}
$$

for all $f \in \operatorname{Dom}(\mathcal{M})$. This yields (2.10). From (2.13) and (2.17) we obtain the equality of the norms (2.11).

Example 2.5. Let $\mathfrak{h}=\mathcal{K}$ and let $g(x)=I$ for all $x$, i.e.,

$$
G f=\int_{\mathbb{R}} f(x) d x, \quad f \in \operatorname{Dom} \mathcal{M} .
$$

Then $G \in \operatorname{Smooth}(\mathcal{M})$ and $\|G\|_{\operatorname{Smooth}(\mathcal{M})}=1$. It is easy to see that $G$ is not closable.

\section{3. $\mathbf{S}_{p}$-VALUED SMOOTHNESS}

\subsection{Definition and characterisation.}

Definition 3.1. For $0<p<\infty$, we write $G \in \operatorname{Smooth}_{p}(H)$, if $G \in \operatorname{Smooth}(H)$ and if for some $C>0$ and for all $\varphi \in L^{2}(\mathbb{R})$,

$$
\|G \varphi(H)\|_{p} \leq C\|\varphi\|_{L^{2}} .
$$

In this case we set

$$
\|G\|_{\operatorname{Smooth}_{p}(H)}=\sup _{\|\varphi\|_{L^{2}}=1}\|G \varphi(H)\|_{p} .
$$

Lemma 3.2. Let $p \geq 2$; then

$$
\|G\|_{\operatorname{Smooth}_{p}(H)}=\sup _{\Lambda \subset \mathbb{R}}|\Lambda|^{-1 / 2}\left\|G E_{H}(\Lambda)\right\|_{p}, \quad p \geq 2,
$$

where the supremum is taken over all finite intervals $\Lambda$. 
Proof. Denote by $A$ the right hand side of (3.1). The inequality $\|G\|_{\operatorname{Smooth}_{p}(H)} \geq A$ follows by taking $\varphi=\mathbb{1}_{\Lambda}$. The converse inequality follows by the same calculation as in the proof of Theorem 2.1, with Schatten norms instead of the operator norms. Indeed, for

$$
\varphi=\sum_{k} \varphi_{k} \mathbb{1}_{\Lambda_{k}}
$$

we have

$$
\begin{array}{r}
\|G \varphi(H)\|_{\mathbf{S}_{p}}^{2}=\left\|G \varphi(H)(G \varphi(H))^{*}\right\|_{\mathbf{S}_{p / 2}} \leq \sum_{k}\left|\varphi_{k}\right|^{2}\left\|G E_{H}\left(\Lambda_{k}\right)\left(G E_{H}\left(\Lambda_{k}\right)\right)^{*}\right\|_{\mathbf{S}_{p / 2}} \\
=\sum_{k}\left|\varphi_{k}\right|^{2}\left\|G E_{H}\left(\Lambda_{k}\right)\right\|_{\mathbf{S}_{p}}^{2} \leq A \sum_{k}\left|\varphi_{k}\right|^{2}\left|\Lambda_{k}\right|=A\|\varphi\|_{L^{2}}^{2},
\end{array}
$$

which gives the required bound.

For $0<p<2$ the argument of Lemma 3.2 is no longer valid, as the triangle inequality fails for the quasi-norm $\|\cdot\|_{p / 2}$.

For $p \geq 2, \mathbf{S}_{p}$-valued smoothness with respect to the multiplication operator is easy to characterise. For $0<p<2$, we have only a necessary condition for $\mathbf{S}_{p}$-valued smoothness.

Theorem 3.3. Let $\mathcal{M}$ be the multiplication operator $(2.9)$ in $\mathcal{H}=L^{2}(\mathbb{R} ; \mathfrak{h})$ and let $G: \mathcal{H} \rightarrow \mathcal{K}$ be an $\mathcal{M}$-bounded operator.

(i) Let $p \geq 2$; then $G \in \operatorname{Smooth}_{p}(\mathcal{M})$ if and only if $G$ can be represented as in (2.10) with some $g \in L^{\infty}\left(\mathbb{R} ; \mathbf{S}_{p}(\mathfrak{h}, \mathcal{K})\right)$. Moreover, in this case we have the equality of the norms

$$
\|g\|_{L^{\infty}\left(\mathbb{R} ; \mathbf{S}_{p}(\mathfrak{h}, \mathcal{K})\right)}=\|G\|_{\operatorname{Smooth}_{p}(\mathcal{M})}, \quad p \geq 2 .
$$

(ii) Let $0<p<2$. If $G \in \operatorname{Smooth}_{p}(\mathcal{M})$, then $G$ can be represented as in (2.10) with some $g \in L^{\infty}\left(\mathbb{R} ; \mathbf{S}_{p}(\mathfrak{h}, \mathcal{K})\right)$ and

$$
\|g\|_{L^{\infty}\left(\mathbb{R} ; \mathbf{S}_{p}(\mathfrak{h}, \mathcal{K})\right)} \leq\|G\|_{\operatorname{Smooth}_{p}(\mathcal{M})}, \quad 0<p<2 .
$$

After the proof of this theorem we will give an example that shows that for $0<p<2$ an operator $G$ represented as in (2.10) with some $g \in L^{\infty}\left(\mathbb{R} ; \mathbf{S}_{p}(\mathfrak{h}, \mathcal{K})\right)$ does not necessarily belong to $\operatorname{Smooth}_{p}(\mathcal{M})$, so one cannot expect equality in (3.2).

We need the following well-known lemma:

Lemma 3.4. Let $\left\{A_{n}\right\}_{n=1}^{\infty}$ be a sequence of non-negative operators which converges *-weakly to an operator $A$. Then

$$
\operatorname{Tr} A \leq \liminf _{n \rightarrow \infty} \operatorname{Tr} A_{n}
$$

(with the understanding that the left side is finite if the right side is). 
Proof. Let $\left\{e_{j}\right\}_{j=1}^{\infty}$ be an orthonormal basis of the underlying Hilbert space. Then for any $J \in \mathbb{N}$,

$$
\sum_{j=1}^{J}\left(A e_{j}, e_{j}\right)=\liminf _{n \rightarrow \infty} \sum_{j=1}^{J}\left(A_{n} e_{j}, e_{j}\right) \leq \liminf _{n \rightarrow \infty} \operatorname{Tr} A_{n} .
$$

The assertion follows as $J \rightarrow \infty$ by monotone convergence.

Proof of Theorem 3.3. Let $g \in L^{\infty}\left(\mathbb{R} ; \mathbf{S}_{p}(\mathfrak{h}, \mathcal{K})\right)$ for some $p \geq 2$ and let $G$ be defined according to (2.10). Then, using (2.12), we obtain

$$
\begin{array}{r}
\left\|G E_{\mathcal{M}}(\Lambda)\right\|_{p}^{2}=\left\|G E_{\mathcal{M}}(\Lambda)\left(G E_{\mathcal{M}}(\Lambda)\right)^{*}\right\|_{p / 2}=\left\|\int_{\Lambda} g(x) g(x)^{*} d x\right\|_{p / 2} \\
\leq\left\|g g^{*}\right\|_{L^{\infty}\left(\mathbb{R} ; \mathbf{S}_{p / 2}(\mathfrak{h}, \mathcal{K})\right)}|\Lambda|=\|g\|_{L^{\infty}\left(\mathbb{R} ; \mathbf{S}_{p}(\mathfrak{h}, \mathcal{K})\right)}^{2}|\Lambda| .
\end{array}
$$

By Lemma 3.2, it follows that $G \in \operatorname{Smooth}_{p}(\mathcal{M})$ and

$$
\|G\|_{\operatorname{Smooth}_{p}(\mathcal{M})} \leq\|g\|_{L^{\infty}\left(\mathbb{R} ; \mathbf{S}_{p}(\mathfrak{h}, \mathcal{K})\right)} .
$$

We now prove the converse implication and assume that $G \in \operatorname{Smooth}_{p}(\mathcal{M})$ for some $p>0$. Since $\operatorname{Smooth}_{p}(\mathcal{M}) \subset \operatorname{Smooth}(\mathcal{M})$, by Theorem 2.4 we have the representation (2.10) with some $g \in L^{\infty}(\mathbb{R} ; \mathcal{B}(\mathfrak{h}, \mathcal{K}))$. We claim that

$$
\left\|\frac{1}{2 \varepsilon} \int_{\lambda-\varepsilon}^{\lambda+\varepsilon} g(x) g(x)^{*} d x-g(\lambda) g(\lambda)^{*}\right\|_{\mathcal{B}(\mathcal{K})} \rightarrow 0 \text { as } \varepsilon \rightarrow 0 \text { for a.e. } \lambda \in \mathbb{R} \text {. }
$$

This is the Lebesgue differentiation theorem for functions on $\mathbb{R}$ valued in the Banach space $\mathcal{B}(\mathcal{K})$; see e.g. [9, Theorem 2.3.4]. Since the function $t \mapsto t^{p / 2}$ is continuous on $[0, \infty)$, we infer that

$$
\left\|\left(\frac{1}{2 \varepsilon} \int_{\lambda-\varepsilon}^{\lambda+\varepsilon} g(x) g(x)^{*} d x\right)^{p / 2}-\left(g(\lambda) g(\lambda)^{*}\right)^{p / 2}\right\| \rightarrow 0 \quad \text { for a.e. } \lambda \in \mathbb{R} .
$$

By the lower semi-continuity of the trace which we have recalled in Lemma 3.4, we obtain for almost every $\lambda \in \mathbb{R}$

$$
\begin{aligned}
&\|g(\lambda)\|_{p}^{p}=\operatorname{Tr}\left(g(\lambda) g(\lambda)^{*}\right)^{p / 2} \leq \liminf _{\epsilon \rightarrow 0} \operatorname{Tr}\left(\frac{1}{2 \varepsilon} \int_{\lambda-\varepsilon}^{\lambda+\varepsilon} g(x) g(x)^{*} d x\right)^{p / 2} \\
&=\liminf _{\epsilon \rightarrow 0}\left\|\frac{1}{2 \varepsilon} G E_{\mathcal{M}}(\lambda-\varepsilon, \lambda+\varepsilon)\left(G E_{\mathcal{M}}(\lambda-\varepsilon, \lambda+\varepsilon)\right)^{*}\right\|_{p / 2}^{p / 2} .
\end{aligned}
$$

By the definition of smoothness with $\varphi=\frac{1}{\sqrt{2 \varepsilon}} \mathbb{1}_{(\lambda-\varepsilon, \lambda+\varepsilon)}$ we have

$$
\left\|\frac{1}{2 \varepsilon} G E_{\mathcal{M}}(\lambda-\varepsilon, \lambda+\varepsilon)\left(G E_{\mathcal{M}}(\lambda-\varepsilon, \lambda+\varepsilon)\right)^{*}\right\|_{p / 2}^{p / 2} \leq\|G\|_{\operatorname{Smooth}_{p}(\mathcal{M})}^{2} .
$$


This implies $g \in L^{\infty}\left(\mathbb{R} ; \mathbf{S}_{p}\right)$ and

$$
\|g\|_{L^{\infty}\left(\mathbb{R} ; \mathbf{S}_{p}\right)} \leq\|G\|_{\operatorname{Smooth}_{p}(\mathcal{M})} .
$$

This completes the proof of the theorem.

Example 3.5. Let $\mathfrak{h}=\mathcal{K}=\ell^{2}$, and let $\left(e_{n}\right)_{n \in \mathbb{N}}$ be the standard basis in $\ell^{2}$. Define

$$
g(x)=\sum_{n=1}^{\infty} \mathbb{1}_{(n-1, n)}(x)\left(\cdot, e_{n}\right) e_{n}, \quad x \in \mathbb{R} .
$$

Then clearly $g \in L^{\infty}\left(\mathbb{R} ; \mathbf{S}_{p}\left(\ell^{2}\right)\right)$ with $\|g\|_{L^{\infty}\left(\mathbb{R} ; \mathbf{S}_{p}\right)}=1$ for any $p>0$. Moreover, for the interval $\Lambda_{N}=(0, N)$ we find similarly to the proof of Theorem 3.3

$$
\left\|G E_{\mathcal{M}}\left(\Lambda_{N}\right)\right\|_{p}^{2}=\left\|\int_{\Lambda_{N}} g(x) g(x)^{*} d x\right\|_{p / 2}=\left\|\sum_{n=1}^{N}\left(\cdot, e_{n}\right) e_{n}\right\|_{p / 2}=N^{2 / p} .
$$

For $0<p<2$ we conclude that

$$
\sup _{\Lambda \subset \mathbb{R}}|\Lambda|^{-1 / 2}\left\|G E_{\mathcal{M}}\left(\Lambda_{N}\right)\right\|_{p} \geq \sup _{N}\left|\Lambda_{N}\right|^{-1 / 2} N^{1 / p}=\infty
$$

and therefore $G \notin \operatorname{Smooth}_{p}(\mathcal{M})$.

\subsection{An interpolation result.}

Lemma 3.6. Let $2<q<\infty$, and let $G \in \operatorname{Smooth}_{q}(H)$. Then there exists a family of operators $G(z): \mathcal{H} \rightarrow \mathcal{K}, 0 \leq \operatorname{Re} z \leq 1$, such that:

(i) $G(z) \in \operatorname{Smooth}(H)$ for all $z$, with $\sup _{0 \leq \operatorname{Re} z \leq 1}\|G(z)\|_{\operatorname{Smooth}(H)}<\infty$;

(ii) $\|G(z)\|_{\operatorname{Smooth}(H)} \leq 1$ for $\operatorname{Re} z=0$;

(iii) $\|G(z)\|_{\text {Smooth }_{2}(H)}^{2} \leq\|G\|_{\operatorname{Smooth}_{q}(H)}^{q}$ for $\operatorname{Re} z=1$;

(iv) $G(2 / q)=G$;

(v) for any $\varphi \in L^{2}(\mathbb{R})$, the family of bounded operators $G(z) \varphi(H)$ is analytic in $z$ for $0<\operatorname{Re} z<1$ and continuous in $z$ for $0 \leq \operatorname{Re} z \leq 1$.

Before coming to the proof, we recall the following consequence of the spectral theorem for self-adjoint operators. Let $H$ be a self-adjoint operator in $\mathcal{H}$; then there exists a Hilbert space $\mathfrak{h}$ and a linear isometry (not necessarily onto)

$$
U: \mathcal{H}^{(\mathrm{ac})}(H) \rightarrow L^{2}(\mathbb{R} ; \mathfrak{h}), \quad \text { such that } \quad \varphi\left(H^{(\mathrm{ac})}\right)=U^{*} \varphi(\mathcal{M}) U,
$$

for any Borel function $\varphi$ on $\mathbb{R}$. Here $\mathcal{M}$ is the multiplication operator (2.9) in $L^{2}(\mathbb{R} ; \mathfrak{h})$. Further, it is easy to see that $G \in \operatorname{Smooth}(H)$ if and only if $G U^{*} \in$ $\operatorname{Smooth}(\mathcal{M})$, with

$$
\|G\|_{\operatorname{Smooth}(H)}=\left\|G U^{*}\right\|_{\operatorname{Smooth}(\mathcal{M})}
$$

and the same is true for the $\operatorname{Smooth}_{q}$ norms. 
Proof of Lemma 3.6. By the above remarks, the question is reduced to the case $H=\mathcal{M}$. By Theorem 3.3, $G$ has the representation

$$
G f=\int_{\mathbb{R}} g(x) f(x) d x
$$

with $g \in L^{\infty}\left(\mathbb{R} ; \mathbf{S}_{q}(\mathfrak{h}, \mathcal{K})\right)$. Write the polar decomposition of $g(x)$ as

$$
g(x)=\omega(x)|g(x)|, \quad x \in \mathbb{R},
$$

where $\omega(x)$ is a partial isometry for a.e. $x \in \mathbb{R}$. Now let us define

$$
G(z) f=\int_{\mathbb{R}} g_{z}(x) f(x) d x, \quad g_{z}(x)=\omega(x)|g(x)|^{q z / 2} .
$$

We have

- $g_{z} \in L^{\infty}(\mathbb{R} ; \mathcal{B})$ for all $z, 0 \leq \operatorname{Re} z \leq 1$, and $\sup _{0 \leq \operatorname{Re} z \leq 1}\left\|g_{z}\right\|_{L^{\infty}(\mathbb{R}, \mathcal{B})}<\infty$;

- $\left\|g_{z}\right\|_{L^{\infty}(\mathbb{R} ; \mathcal{B})} \leq 1$ for $\operatorname{Re} z=0$;

- $\left\|g_{z}\right\|_{L^{\infty}\left(\mathbb{R} ; \mathbf{S}_{2}\right)}^{2}=\|g\|_{L^{\infty}\left(\mathbb{R} ; \mathbf{S}_{q}\right)}^{q}$ for $\operatorname{Re} z=1$;

- $g_{2 / q}=g$.

From here, again using Theorem 3.3, we obtain the properties (i)-(iii) of $G(z)$. The property (iv) is obvious from the definition, and the property (v) is straightforward to check.

\section{Double operator integrals}

4.1. Overview. The notion of double operator integrals (DOI) was initially introduced by Daletskii and Krein in [4] and developed by Birman and Solomyak in [2] (see [3] for a modern account of the theory and for further historical references). Here we consider DOI from a different viewpoint; essentially, we construct an alternative version of the theory of DOI under a different set of assumptions.

Throughout this section, $H_{0}$ and $H_{1}$ are self-adjoint operators in $\mathcal{H}$ and $G_{0}, G_{1}$ are operators from $\mathcal{H}$ to $\mathcal{K}$ such that $G_{0} \in \operatorname{Smooth}\left(H_{0}\right)$ and $G_{1} \in \operatorname{Smooth}\left(H_{1}\right)$. We will work with bounded operators $a$ on $L^{2}(\mathbb{R})$ and with their integral kernels $a(x, y)$. (In practice, we will only need the notion of an integral kernel for finite rank operators $a$; in this case this notion can be unambiguously defined without difficulty.) Informally speaking, we would like to define the double operator integral

$$
\operatorname{DOI}(a)=\int_{\mathbb{R}} \int_{\mathbb{R}} a(x, y) d E_{H_{1}}(x) G_{1}^{*} G_{0} d E_{H_{0}}(y),
$$

initially for finite rank operators $a$ and eventually for all bounded operators $a$ on $L^{2}(\mathbb{R})$. In other words, for fixed $G_{0}, G_{1}, H_{0}, H_{1}$, we consider the map

$$
\text { DOI }: \mathcal{B}\left(L^{2}(\mathbb{R})\right) \rightarrow \mathcal{B}(\mathcal{H})
$$

defined initially on the set of all finite rank operators $a$. We prove that this map can be extended in a natural way to the whole space $\mathcal{B}\left(L^{2}(\mathbb{R})\right)$, that it is bounded 
and satisfies the operator norm and the Schatten norm bounds

$$
\begin{aligned}
\|\operatorname{DOI}(a)\|_{\mathcal{B}} & \leq\left\|G_{0}\right\|_{\operatorname{Smooth}\left(H_{0}\right)}\left\|G_{1}\right\|_{\operatorname{Smooth}\left(H_{1}\right)}\|a\|_{\mathcal{B}}, \\
\|\operatorname{DOI}(a)\|_{p} & \leq\left\|G_{0}\right\|_{\operatorname{Smooth}_{q}\left(H_{0}\right)}\left\|G_{1}\right\|_{\operatorname{Smooth}_{r}\left(H_{1}\right)}\|a\|_{p}, \quad \frac{1}{p}=\frac{1}{q}+\frac{1}{r} .
\end{aligned}
$$

In order to make sense of the integral (4.1), in the standard approach to the theory of double operator integrals [2, 3] one has to assume some degree of regularity of the kernel $a(x, y)$. In our framework, the regularity of $a(x, y)$ is not needed, as we are using the smoothness of $G_{0}$ and $G_{1}$ instead.

Recall that if $G \in \operatorname{Smooth}(H)$, then $\left.G\right|_{\mathcal{H}^{(\text {sing })(H)}}=0$. Thus, it is natural to define $\operatorname{DOI}(a)$ such that it satisfies the property

$$
(\operatorname{DOI}(a) u, v)=0 \quad \text { if } u \in \mathcal{H}^{(\operatorname{sing})}\left(H_{0}\right) \text { or } v \in \mathcal{H}^{(\text {sing })}\left(H_{1}\right)
$$

(or both). Thus, essentially $\operatorname{DOI}(a)$ acts from $\mathcal{H}^{(\mathrm{ac})}\left(H_{0}\right)$ to $\mathcal{H}^{(\mathrm{ac})}\left(H_{1}\right)$.

It will be convenient to use the following notation for the constants in the estimates (4.2) and (4.3):

$$
A:=\left\|G_{0}\right\|_{\operatorname{Smooth}\left(H_{0}\right)}\left\|G_{1}\right\|_{\operatorname{Smooth}\left(H_{1}\right)}, \quad A_{q, r}:=\left\|G_{0}\right\|_{\operatorname{Smooth}_{q}\left(H_{0}\right)}\left\|G_{1}\right\|_{\operatorname{Smooth}_{r}\left(H_{1}\right)} .
$$

4.2. $\operatorname{DOI}(a)$ for finite rank $a$. We begin by defining $\mathrm{DOI}(a)$ for finite rank operators $a$. Let $a$ be given by its Schmidt series,

$$
a=\sum_{n=1}^{N} s_{n}\left(\cdot, \varphi_{n}\right) \psi_{n}
$$

where $N$ is finite, $\left\{s_{n}\right\}$ are the singular values of $a$ and $\left\{\varphi_{n}\right\},\left\{\psi_{n}\right\}$ are orthonormal sets. Then the integral kernel of $a$ is given by

$$
a(x, y)=\sum_{n=1}^{N} s_{n} \psi_{n}(x) \overline{\varphi_{n}(y)}, \quad x, y \in \mathbb{R} .
$$

In this case, we set

$$
\operatorname{DOI}(a)=\sum_{n=1}^{N} s_{n}\left(G_{1} \psi_{n}\left(H_{1}\right)^{*}\right)^{*} G_{0} \varphi_{n}\left(H_{0}\right)^{*} .
$$

From this definition it follows, in particular, that the property (4.4) is satisfied.

First we need to check that definition (4.7) is independent of the choice of the Schmidt series representation (4.6). This will follow from the next lemma.

Lemma 4.1. For $j=0,1$, let $U_{j}$ be a diagonalization isometry as in (3.3), i.e.

$$
U_{j}: \mathcal{H}^{(a c)}\left(H_{j}\right) \rightarrow L^{2}(\mathbb{R} ; \mathfrak{h}), \quad H_{j}=U_{j}^{*} \mathcal{M} U_{j},
$$


where $\mathfrak{h}$ is a Hilbert space and $\mathcal{M}$ is the operator of multiplication by the independent variable in $L^{2}(\mathbb{R} ; \mathfrak{h})$. For $v_{j} \in \mathcal{H}^{(a c)}\left(H_{j}\right)$, denote $\widehat{v}_{j}=U_{j} v_{j} \in L^{2}(\mathbb{R} ; \mathfrak{h})$ and write the representation of Theorem 2.4 for $G_{j} U_{j}^{*}$ as

$$
G_{j} U_{j}^{*} \widehat{v}_{j}=\int_{\mathbb{R}} g_{j}(x) \widehat{v}_{j}(x) d x, \quad g_{j} \in L^{\infty}(\mathbb{R} ; \mathcal{B}(\mathfrak{h}, \mathcal{K})), \quad j=0,1 .
$$

Then for all finite rank operators a, we have

$$
\left(\operatorname{DOI}(a) v_{0}, v_{1}\right)=\int_{\mathbb{R}} \int_{\mathbb{R}} a(x, y)\left(g_{0}(y) \widehat{v}_{0}(y), g_{1}(x) \widehat{v}_{1}(x)\right)_{\mathcal{K}} d x d y .
$$

Proof. By linearity, it suffices to prove (4.8) for rank one operators $a$. Let $a(x, y)=$ $\psi(x) \overline{\varphi(y)}$. Then

$$
\begin{aligned}
\left(\operatorname{DOI}(a) v_{0}, v_{1}\right)= & \left(G_{0} \varphi\left(H_{0}\right)^{*} v_{0}, G_{1} \psi\left(H_{1}\right)^{*} v_{1}\right)_{\mathcal{K}} \\
& =\left(G_{0} U_{0}^{*} \varphi(\mathcal{M})^{*} U_{0} v_{0}, G_{1} U_{1}^{*} \psi(\mathcal{M})^{*} U_{1} v_{1}\right)_{\mathcal{K}} \\
= & \left(\int_{\mathbb{R}} g_{0}(y) \overline{\varphi(y)} \widehat{v}_{0}(y) d y, \int_{\mathbb{R}} g_{1}(x) \overline{\psi(x)} \widehat{v}_{1}(x) d x\right)_{\mathcal{K}} \\
= & \int_{\mathbb{R}} \int_{\mathbb{R}} \psi(x) \overline{\varphi(y)}\left(g_{0}(y) \widehat{v}_{0}(y), g_{1}(x) \widehat{v}_{1}(x)\right)_{\mathcal{K}} d x d y
\end{aligned}
$$

as required.

This lemma shows that $\operatorname{DOI}(a)$ can be alternatively defined through the integral kernel of $a$. Since the integral kernel is independent of the choice of the Schmidt series representation (4.6), our definition of $\operatorname{DOI}(a)$ is also independent of this choice.

Lemma 4.2. For any finite rank operator a, one has (with $A$ as in (4.5))

$$
\|\operatorname{DOI}(a)\|_{\mathcal{B}} \leq A\|a\|_{\mathcal{B}}
$$

Proof. Let $a$ be as in (4.6); observe that $\max _{n} s_{n}=\|a\|_{\mathcal{B}}$. The sesquilinear form of $\operatorname{DOI}(a)$ is

$$
\left(\operatorname{DOI}(a) v_{0}, v_{1}\right)=\sum_{n=1}^{N} s_{n}\left(G_{0} \varphi_{n}\left(H_{0}\right)^{*} v_{0}, G_{1} \psi_{n}\left(H_{1}\right)^{*} v_{1}\right) .
$$

Applying Cauchy-Schwarz and Theorem 2.2, we can estimate this form as follows:

$$
\begin{aligned}
& \left|\left(\operatorname{DOI}(a) v_{0}, v_{1}\right)\right| \leq \sum_{n=1}^{N} s_{n}\left\|G_{0} \varphi_{n}\left(H_{0}\right)^{*} v_{0}\right\|_{\mathcal{K}}\left\|G_{1} \psi_{n}\left(H_{1}\right)^{*} v_{1}\right\|_{\mathcal{K}} \\
\leq & \|a\|_{\mathcal{B}}\left(\sum_{n=1}^{N}\left\|G_{0} \varphi_{n}\left(H_{0}\right)^{*} v_{0}\right\|_{\mathcal{K}}^{2}\right)^{1 / 2}\left(\sum_{n=1}^{N}\left\|G_{1} \psi_{n}\left(H_{1}\right)^{*} v_{1}\right\|_{\mathcal{K}}^{2}\right)^{1 / 2} \leq A\|a\|_{\mathcal{B}}\left\|v_{0}\right\|_{\mathcal{H}}\left\|v_{1}\right\|_{\mathcal{H}},
\end{aligned}
$$

as required. 
Lemma 4.3. Let $a_{n}$, a be finite rank operators such that $a_{n} \rightarrow a *$-weakly. Then $\operatorname{DOI}\left(a_{n}\right) \rightarrow \operatorname{DOI}(a) *$-weakly.

Proof. By linearity it suffices to consider the case $a_{n} \rightarrow 0 *$-weakly. By Lemma 4.1, we have

$$
\left(\operatorname{DOI}\left(a_{n}\right) v_{0}, v_{1}\right)=\int_{\mathbb{R}} \int_{\mathbb{R}} a_{n}(x, y)\left(g_{0}(y) \widehat{v}_{0}(y), g_{1}(x) \widehat{v}_{1}(x)\right)_{\mathcal{K}} d x d y,
$$

where

$$
\int_{\mathbb{R}}\left\|g_{j}(x) \widehat{v}_{j}(x)\right\|_{\mathcal{K}}^{2} d x \leq\left\|G_{j}\right\|_{\operatorname{Smooth}\left(H_{j}\right)}^{2}\left\|v_{j}\right\|_{\mathcal{H}}^{2}, \quad j=0,1 .
$$

Let $\left\{e_{\ell}\right\}$ be an orthonormal basis in $\mathcal{K}$. Denote

$$
F_{j, \ell}(x)=\left(g_{j}(x) \widehat{v}_{j}(x), e_{\ell}\right), \quad x \in \mathbb{R}, \quad j=0,1,
$$

and consider the operator $K$ in $L^{2}(\mathbb{R})$ with the integral kernel

$$
K(x, y)=\sum_{\ell} F_{0, \ell}(x) \overline{F_{1, \ell}(y)}
$$

This operator is trace class, because

$\sum_{\ell}\left\|F_{0, \ell}\right\|_{L^{2}}\left\|F_{1, \ell}\right\|_{L^{2}} \leq\left(\sum_{\ell}\left\|F_{0, \ell}\right\|_{L^{2}}^{2}\right)^{1 / 2}\left(\sum_{\ell}\left\|F_{1, \ell}\right\|_{L^{2}}^{2}\right)^{1 / 2} \leq\left\|g_{0} \widehat{v}_{0}\right\|_{L^{2}}\left\|g_{1} \widehat{v}_{1}\right\|_{L^{2}}<\infty$.

Now let us expand the inner product in (4.10) as

$$
\left(g_{0}(y) \widehat{v}_{0}(y), g_{1}(x) \widehat{v}_{1}(x)\right)_{\mathcal{K}}=\sum_{\ell}\left(g_{0}(y) \widehat{v}_{0}(y), e_{\ell}\right)_{\mathcal{K}}\left(e_{\ell}, g_{1}(x) \widehat{v}_{1}(x)\right)_{\mathcal{K}} ;
$$

this yields

$$
\left(\operatorname{DOI}\left(a_{n}\right) v_{0}, v_{1}\right)=\sum_{\ell} \int_{\mathbb{R}} \int_{\mathbb{R}} a_{n}(x, y) F_{0, \ell}(y) \overline{F_{1, \ell}(x)} d x d y=\operatorname{Tr}\left(a_{n} K\right) .
$$

By our assumption on $*$-weak convergence, we have $\operatorname{Tr}\left(a_{n} K\right) \rightarrow 0$ as $n \rightarrow \infty$, and therefore $\operatorname{DOI}\left(a_{n}\right) \rightarrow 0 *$-weakly.

4.3. $\operatorname{DOI}(a)$ for bounded and compact $a$. In the previous subsection, we have defined the map

$$
\mathrm{DOI}: \mathcal{B}\left(L^{2}(\mathbb{R})\right) \rightarrow \mathcal{B}(\mathcal{H})
$$

on the set of all finite rank operators; we have checked this map is bounded in the operator norm and continuous with respect to the $*$-weak convergence. Since finite rank operators are $*$-weakly dense in the set of bounded operators, we can extend this map (by $*$-weak continuity) onto the whole set $\mathcal{B}\left(L^{2}(\mathbb{R})\right)$.

Lemma 4.4. The map (4.11), extended as explained above, is bounded with respect to the operator norm, and the operator norm bound (4.2) holds true. The property (4.4) also holds for any bounded a. 
Proof. Let $P_{n}$ be a sequence of finite rank orthogonal projections in $L^{2}(\mathbb{R})$ such that $P_{n} \rightarrow I$ strongly as $n \rightarrow \infty$. Denote $a_{n}=P_{n} a P_{n}$. Then $a_{n} \rightarrow a *$-weakly and $\left\|a_{n}\right\|_{\mathcal{B}} \leq\|a\|_{\mathcal{B}}$ for all $n$. Using the bound (4.9) for finite rank operators, we obtain

$$
\|\operatorname{DOI}(a)\|_{\mathcal{B}} \leq \liminf _{n \rightarrow \infty}\left\|\operatorname{DOI}\left(a_{n}\right)\right\|_{\mathcal{B}} \leq A \liminf _{n \rightarrow \infty}\left\|a_{n}\right\|_{\mathcal{B}} \leq A\|a\|_{\mathcal{B}}
$$

Finally, it is clear that the property (4.4) is preserved under the weak limits.

Recall that the class $\operatorname{Smooth}_{\infty}(H) \subset \operatorname{Smooth}(H)$ is defined by the additional compactness assumption (2.8).

Lemma 4.5. Assume that $a \in \mathbf{S}_{\infty}, G_{0} \in \operatorname{Smooth}\left(H_{0}\right)$ and $G_{1} \in \operatorname{Smooth}\left(H_{1}\right)$, and suppose in addition that either $G_{0} \in \operatorname{Smooth}_{\infty}\left(H_{0}\right)$ or $G_{1} \in \operatorname{Smooth}_{\infty}\left(H_{1}\right)$ (or both). Then $\operatorname{DOI}(a) \in \mathbf{S}_{\infty}$.

Proof. Consider the case $G_{0} \in \operatorname{Smooth}_{\infty}\left(H_{0}\right)$. By Lemma 4.4, it suffices to check that $\operatorname{DOI}(a) \in \mathbf{S}_{\infty}$ for all finite rank $a$. By linearity, it suffices to consider rank one operators $a$. Let $a(x, y)=\psi(x) \overline{\varphi(y)}$; then

$$
\operatorname{DOI}(a)=\left(G_{1} \psi\left(H_{1}\right)^{*}\right)^{*} G_{0} \varphi\left(H_{0}\right)^{*} .
$$

Here $G_{1} \psi\left(H_{1}\right)^{*}$ is bounded by Theorem 2.1 and $G_{0} \varphi\left(H_{0}\right)^{*}$ is compact by Lemma 2.3. This gives the compactness of DOI $(a)$. The case $G_{1} \in \operatorname{Smooth}_{\infty}\left(H_{1}\right)$ is considered in the same way.

4.4. $\operatorname{DOI}(a)$ for $a \in \mathbf{S}_{p}$.

Theorem 4.6. Let $p, q, r$ be finite positive numbers such that $\frac{1}{p}=\frac{1}{q}+\frac{1}{r}$. Let $G_{0} \in \operatorname{Smooth}_{q}\left(H_{0}\right), G_{1} \in \operatorname{Smooth}_{r}\left(H_{1}\right)$. Then for all $a \in \mathbf{S}_{p}$, we have $\operatorname{DOI}(a) \in \mathbf{S}_{p}$ and the Schatten norm bound (4.3) holds true.

This extends to $q=\infty$ (resp. $r=\infty$ ) if one replaces $\operatorname{Smooth}_{q}\left(H_{0}\right)$ (resp. $\left.\operatorname{Smooth}_{r}\left(H_{1}\right)\right)$ by $\operatorname{Smooth}\left(H_{0}\right)$ (resp. $\left.\operatorname{Smooth}\left(H_{1}\right)\right)$.

Proof. First let us consider the case of finite $q, r$. By a density argument it suffices to prove (4.3) for finite rank operators $a$. Let $a$ be given by its Schmidt series (4.6), so

$$
\|a\|_{p}^{p}=\sum_{n=1}^{N} s_{n}^{p} \quad \text { and } \quad \operatorname{DOI}(a)=\sum_{n=1}^{N} s_{n}\left(G_{1} \psi_{n}\left(H_{1}\right)^{*}\right)^{*} G_{0} \varphi_{n}\left(H_{0}\right)^{*} .
$$

We write $\operatorname{DOI}(a)$ in a factorised form:

$$
\operatorname{DOI}(a)=T_{1}^{*} T_{0}
$$

where the maps $T_{j}: \mathcal{H} \rightarrow \ell^{2}(\mathbb{N} ; \mathcal{K}), j=0,1$ are defined by

$$
\begin{array}{ll}
\left(T_{0} u\right)_{n}=s_{n}^{p / q} G_{0} \varphi_{n}\left(H_{0}\right)^{*} u, & n \in \mathbb{N}, \\
\left(T_{1} u\right)_{n}=s_{n}^{p / r} G_{1} \psi_{n}\left(H_{1}\right)^{*} u, & n \in \mathbb{N} .
\end{array}
$$


Our aim is to show that $T_{0} \in \mathbf{S}_{q}$ and $T_{1} \in \mathbf{S}_{r}$ with the norm bounds

$$
\begin{aligned}
\left\|T_{0}\right\|_{q} & \leq\|a\|_{p}^{p / q}\left\|G_{0}\right\|_{\operatorname{Smooth}_{q}\left(H_{0}\right)}, \\
\left\|T_{1}\right\|_{r} & \leq\|a\|_{p}^{p / r}\left\|G_{1}\right\|_{\operatorname{Smooth}_{r}\left(H_{1}\right)} .
\end{aligned}
$$

From (4.12) and (4.13) the required result follows immediately by an application of the "Hölder inequality for $\mathbf{S}_{p}$ classes":

$$
\|\operatorname{DOI}(a)\|_{p}=\left\|T_{1}^{*} T_{0}\right\|_{p} \leq\left\|T_{1}\right\|_{q}\left\|T_{0}\right\|_{r} \leq\|a\|_{p} A_{q, r} .
$$

Let us prove the bound (4.12); the second bound (4.13) is considered in the same way.

Case 1: $0<q \leq 2$. Consider the operator

$$
T_{0}^{*} T_{0}=\sum_{n=1}^{N} s_{n}^{2 p / q}\left(G_{0} \varphi_{n}\left(H_{0}\right)^{*}\right)^{*} G_{0} \varphi_{n}\left(H_{0}\right)^{*}
$$

We use the "triangle inequality" for $\|\cdot\|_{q / 2}^{q / 2}$, see (1.12):

$$
\left\|T_{0}\right\|_{q}^{q}=\left\|T_{0}^{*} T_{0}\right\|_{q / 2}^{q / 2} \leq \sum_{n=1}^{N} s_{n}^{p}\left\|\left(G_{0} \varphi_{n}\left(H_{0}\right)^{*}\right)^{*} G_{0} \varphi_{n}\left(H_{0}\right)^{*}\right\|_{q / 2}^{q / 2}=\sum_{n=1}^{N} s_{n}^{p}\left\|G_{0} \varphi_{n}\left(H_{0}\right)^{*}\right\|_{q}^{q} .
$$

By the definition of the $\mathbf{S}_{q}$-valued smoothness,

$$
\left\|G_{0} \varphi_{n}\left(H_{0}\right)^{*}\right\|_{q} \leq\left\|G_{0}\right\|_{\operatorname{Smooth}_{q}\left(H_{0}\right)}\left\|\varphi_{n}\right\|_{L^{2}}=\left\|G_{0}\right\|_{\operatorname{Smooth}_{q}\left(H_{0}\right)}
$$

since $\varphi_{n}$ are normalised in $L^{2}$. Putting this together, we obtain the bound (4.12).

Case 2: $q \geq 2$. Here we use complex interpolation between the cases $q=2$ and $q=\infty$ and employ Lemma 3.6.

Let $G_{0}(z)$ be the analytic family as in Lemma 3.6 with $G=G_{0}$ and $H=H_{0}$. For $0 \leq \operatorname{Re} z \leq 1$, let $T_{0}(z): \mathcal{H} \rightarrow \ell^{2}(\mathbb{N} ; \mathcal{K})$ be defined by

$$
\left(T_{0}(z) u\right)_{n}=s_{n}^{p z / 2} G_{0}(z) \varphi_{n}\left(H_{0}\right)^{*} u, \quad n \geq 1 .
$$

Let us compute the operator norm of $T_{0}(z)$. Using Theorem 2.2, we obtain

$$
\begin{aligned}
\sum_{n=1}^{N}\left\|\left(T_{0}(z) u\right)_{n}\right\|_{\mathcal{K}}^{2} \leq\|a\|_{\mathcal{B}}^{p \operatorname{Re} z} \sum_{n=1}^{N}\left\|G_{0}(z) \varphi_{n}\left(H_{0}\right)^{*} u\right\|_{\mathcal{K}}^{2} & \\
& \leq\|a\|_{\mathcal{B}}^{p \operatorname{Re} z}\left\|G_{0}(z)\right\|_{\operatorname{Smooth}\left(H_{0}\right)}^{2}\|u\|_{\mathcal{H}}^{2} .
\end{aligned}
$$

Thus, $T_{0}(z)$ is bounded in the operator norm for all $0 \leq \operatorname{Re} z \leq 1$ and

$$
\left\|T_{0}(z)\right\|_{\mathcal{B}} \leq\left\|G_{0}(z)\right\|_{\operatorname{Smooth}\left(H_{0}\right)} \leq 1, \quad \operatorname{Re} z=0 .
$$


Next, for $\operatorname{Re} z=1$ the operator $T_{0}(z)$ is Hilbert-Schmidt. Indeed, using the estimates of Lemma 3.6, we obtain

$$
\begin{aligned}
\left\|T_{0}(z)\right\|_{2}^{2}=\sum_{n=1}^{N} s_{n}^{p}\left\|G_{0}(z) \varphi_{n}\left(H_{0}\right)^{*}\right\|_{2}^{2} & \\
& \leq \sum_{n=1}^{N} s_{n}^{p}\left\|G_{0}(z)\right\|_{\operatorname{Smooth}_{2}\left(H_{0}\right)}^{2} \leq\|a\|_{p}^{p}\left\|G_{0}\right\|_{\operatorname{Smooth}_{q}\left(H_{0}\right)}^{q}, \quad \operatorname{Re} z=1 .
\end{aligned}
$$

Further, it is straighforward to see that $T_{0}(z)$ is analytic in $0<\operatorname{Re} z<1$, operator norm continuous for $0 \leq \operatorname{Re} z \leq 1$ and $T_{0}(2 / q)=T_{0}$. By Hadamard's three lines theorem for Schatten classes [8, Thm. III.13.1], we obtain

$$
\left\|T_{0}\right\|_{q}=\left\|T_{0}(2 / q)\right\|_{q} \leq\left(\|a\|_{p}^{p / 2}\left\|G_{0}\right\|_{\operatorname{Smooth}_{q}\left(H_{0}\right)}^{q / 2}\right)^{2 / q}=\|a\|_{p}^{p / q}\left\|G_{0}\right\|_{\operatorname{Smooth}_{q}\left(H_{0}\right)},
$$

as required.

Finally, let us briefly discuss the case $r=\infty, q=p$ (the case $q=\infty, r=p$ is considered in the same way). Here we set

$$
\begin{aligned}
& \left(T_{0} u\right)_{n}=s_{n} G_{0} \varphi_{n}\left(H_{0}\right)^{*} u, \quad n \in \mathbb{N}, \\
& \left(T_{1} u\right)_{n}=G_{1} \psi_{n}\left(H_{1}\right)^{*} u, \quad n \in \mathbb{N} .
\end{aligned}
$$

Now we have an operator norm bound for $T_{1}$ by Theorem 2.2 and the $\mathbf{S}_{q}$-norm bound for $T_{0}$ by the same argument as above (considering separately the $q \leq 2$ and $q \geq 2$ cases). Combining these bounds, we obtain

$$
\|\operatorname{DOI}(a)\|_{p}=\left\|T_{1}^{*} T_{0}\right\|_{p} \leq\left\|T_{1}\right\|_{\mathcal{B}}\left\|T_{0}\right\|_{p} \leq\|a\|_{p} A_{p, \infty},
$$

as required.

\section{THE MAP $f \mapsto \check{f}$}

5.1. Overview. As in the Introduction, for a function $f: \mathbb{R} \rightarrow \mathbb{C}$, we denote by $\check{f}$ the divided difference

$$
\check{f}(x, y):=\frac{f(x)-f(y)}{x-y}, \quad x, y \in \mathbb{R} .
$$

By a slight abuse of notation, we also denote by $\breve{f}$ the operator in $L^{2}(\mathbb{R})$ with the integral kernel $\breve{f}(x, y)$. Of course, this definition requires some assumptions on $f$; we will be more precise below. Our aim in this section is to establish the boundedness of $f \mapsto \breve{f}$ as a map from $\operatorname{BMO}(\mathbb{R})$ to $\mathcal{B}\left(L^{2}(\mathbb{R})\right)$ and from $B_{p, p}^{1 / p}(\mathbb{R})$ to $\mathbf{S}_{p}$. The content of this section is probably well-known to specialists; we just need to recall the required results in notation convenient for the next section. 
5.2. Preliminaries on BMO. The Hardy space $H^{p}\left(\mathbb{C}_{+}\right), p \geq 1$, is defined in the standard way as the space of all analytic functions $u$ in the upper half-plane such that the norm

$$
\|u\|_{H^{p}\left(\mathbb{C}_{+}\right)}^{p}=\sup _{y>0} \int_{-\infty}^{\infty}|u(x+i y)|^{p} d x
$$

is finite. As usual, we identify the function $u \in H^{p}\left(\mathbb{C}_{+}\right)$with its boundary values $u(x)=u(x+i 0)$, which exist for a.e. $x \in \mathbb{R}$. The spaces $H^{p}\left(\mathbb{C}_{-}\right)$are defined analogously. In fact, we will only need the cases $p=1$ and $p=2$.

The space $\mathrm{BMO}(\mathbb{R})$ (bounded mean oscillation) consists of all locally integrable functions $f$ on $\mathbb{R}$ such that the following supremum over all bounded intervals $I \subset \mathbb{R}$ is finite:

$$
\sup _{I}\left\langle\left|f-\langle f\rangle_{I}\right|\right\rangle_{I}<\infty, \quad\langle f\rangle_{I}=|I|^{-1} \int_{I} f(x) d x .
$$

Observe that this supremum vanishes on constant functions. Strictly speaking, the elements of $\mathrm{BMO}(\mathbb{R})$ should be regarded not as functions but as equivalence classes $\{f+$ const $\}$; in practice, we will deal with individual functions but bear in mind that an arbitrary constant can be added to a function without affecting the BMO norm. Observe that for constant functions, the kernel (1.7) vanishes identically.

Functions in $\operatorname{BMO}(\mathbb{R})$ belong to $L^{p}(-R, R)$ for any $R>0$ and any $p<\infty$, but not for $p=\infty$ : they may have logarithmic singularities. These functions also satisfy [7, Theorem VI.1.2]

$$
f \in \operatorname{BMO}(\mathbb{R}) \Rightarrow \int_{-\infty}^{\infty} \frac{|f(x)|}{1+x^{2}} d x<\infty .
$$

Fefferman's duality theorem [7, Theorem VI.4.4] says that for any $f \in \operatorname{BMO}(\mathbb{R})$, the linear functional on $H^{1}\left(\mathbb{C}_{+}\right)$,

$$
T_{f}(u):=\int_{-\infty}^{\infty} f(x) u(x) d x,
$$

defined initially on a suitable dense set of functions $u$, extends to the whole space $H^{1}\left(\mathbb{C}_{+}\right)$as a bounded linear functional and that conversely, any bounded linear functional on $H^{1}\left(\mathbb{C}_{+}\right)$can be realised in this way with some $f \in \operatorname{BMO}(\mathbb{R})$. The norm of $T_{f}$ in the dual space $H^{1}\left(\mathbb{C}_{+}\right)^{*}$ will be denoted by $\left\|T_{f}\right\|_{H^{1}\left(\mathbb{C}_{+}\right)^{*} \text {. }}$

A minor technical issue here is that the integral in (5.2) need not make sense for all $f \in \mathrm{BMO}$ and all $u \in H^{1}\left(\mathbb{C}_{+}\right)$. This explains the need for using certain dense sets of $f$ 's and $u$ 's in what follows.

There are many equivalent ways to define a norm on $\operatorname{BMO}(\mathbb{R})$. We choose the one directly related to Fefferman's duality theorem. For $f \in \operatorname{BMO}(\mathbb{R})$, we set

$$
\|f\|_{\mathrm{BMO}}:=\max \left\{\left\|T_{f}\right\|_{H^{1}\left(\mathbb{C}_{+}\right)^{*}},\left\|T_{\bar{f}}\right\|_{H^{1}\left(\mathbb{C}_{+}\right)^{*}}\right\} .
$$

We will say that $f_{n} \rightarrow f *$-weakly in $\operatorname{BMO}(\mathbb{R})$, if we have the weak convergence of linear functionals $T_{f_{n}} \rightarrow T_{f}$ and $T_{\bar{f}_{n}} \rightarrow T_{\bar{f}}$ on $H^{1}\left(\mathbb{C}_{+}\right)$. 
We will denote by $\mathcal{R}$ the set of all bounded rational functions of $x \in \mathbb{R}$ :

$$
\mathcal{R}=\{p / q: p, q \text { polynomials, } \operatorname{deg} p \leq \operatorname{deg} q, q(x) \neq 0 \text { for } x \in \mathbb{R}\} .
$$

The subspace $\mathrm{CMO}(\mathbb{R}) \subset \mathrm{BMO}(\mathbb{R})$ (continuous mean oscillation) is the closure of all rational functions $\mathcal{R}$ in $\mathrm{BMO}(\mathbb{R})$. (Alternatively, one can define $\mathrm{CMO}$ as the closure in BMO of the set of all functions of the form $f+$ const, $f \in C_{0}^{\infty}(\mathbb{R})$.)

Remark. The space $\mathrm{CMO}(\mathbb{R})$ is slightly smaller than the more commonly used space $\operatorname{VMO}(\mathbb{R})$ (vanishing mean oscillation) of functions defined by the condition

$$
\lim _{\epsilon \rightarrow 0} \sup _{|I| \leq \epsilon}\left\langle\left|f-\langle f\rangle_{I}\right|\right\rangle_{I}=0
$$

(see, e.g., [18, Section 2A]). Roughly speaking, the functions in VMO must be "more regular than BMO" locally, while the functions in CMO must be "more regular than BMO" both locally and at infinity. For example, the function $\log (1+$ $x^{2}$ ) belongs to $\mathrm{VMO}$ but not to CMO.

Finally, we will need the following

Lemma 5.1. The set $\mathcal{R}$ of rational functions is dense in $\mathrm{BMO}(\mathbb{R})$ with respect to *-weak convergence.

The proof is given in the Appendix.

5.3. Besov spaces. Let $w \in C_{0}^{\infty}(\mathbb{R}), w \geq 0$, be a function with $\operatorname{supp} w \subset[1 / 2,2]$ and such that

$$
\sum_{j \in \mathbb{Z}} w_{j}(x)=1, \quad x>0, \quad \text { where } w_{j}(x)=w\left(x / 2^{j}\right) .
$$

The (homogeneous) Besov class $B_{p, p}^{1 / p}(\mathbb{R})$ is defined as the space of tempered distributions $f$ on $\mathbb{R}$ such that

$$
\|f\|_{B_{p, p}^{1 / p}}^{p}:=\sum_{j \in \mathbb{Z}} 2^{j}\left(\left\|f * \widehat{w}_{j}\right\|_{L^{p}(\mathbb{R})}^{p}+\| f *{\widehat{\widehat{w}_{j}}}_{L^{p}(\mathbb{R})}^{p}\right)<\infty .
$$

Here $*$ is the convolution and $\widehat{w}_{j}$ is the Fourier transform of $w_{j}$,

$$
\widehat{w}_{j}(t)=\frac{1}{2 \pi} \int_{\mathbb{R}} e^{-i t x} w_{j}(x) d x .
$$

Observe that according to this definition, any polynomial $f$ belongs to $B_{p, p}^{1 / p}(\mathbb{R})$ (as the Fourier transform of a polynomial is supported at the origin). In the context of this paper, we consider $B_{p, p}^{1 / p}(\mathbb{R}) \cap \mathrm{BMO}(\mathbb{R})$, which reduces an arbitrary polynomial to an arbitrary constant.

The definition of $B_{p, p}^{1 / p}$ is independent of the choice of the function $w$. However, the precise value of $\|f\|_{B_{p, p}^{1 / p}}$ will, of course, depend on this choice. 
5.4. Discussion: $\check{f}$ and Hankel operators. Recall that the orthogonal projection $P_{+}: L^{2}(\mathbb{R}) \rightarrow H^{2}\left(\mathbb{C}_{+}\right)$onto the Hardy class is given by

$$
\left(P_{+} u\right)(x)=-\frac{1}{2 \pi i} \lim _{\varepsilon \rightarrow+0} \int_{-\infty}^{\infty} \frac{u(y)}{x-y+i \varepsilon} d y, \quad u \in L^{2}(\mathbb{R}) .
$$

Comparing this with (1.7), we see that, at least for smooth bounded functions $f$, the operator $\breve{f}$ can be identified with the commutator $2 \pi i\left[P_{+}, M_{f}\right]$, where $M_{f}$ is the operator of multiplication by $f$. Further, formally we have (denoting $P_{-}=I-P_{+}$)

$$
\frac{1}{2 \pi i} \check{f}=P_{+} M_{f}-M_{f} P_{+}=P_{+} M_{f} P_{-}-P_{-} M_{f} P_{+} .
$$

In accordance with this, we define $\breve{f}$ initially via the sesquilinear form (denoting $\left.u_{ \pm}=P_{ \pm} u\right)$

$$
(\check{f} u, v)=2 \pi i\left(f u_{-}, v_{+}\right)-2 \pi i\left(f u_{+}, v_{-}\right), \quad u, v \in L_{\text {comp }}^{\infty}(\mathbb{R}) .
$$

Let us explain why the inner products in (5.4) are well-defined. Since $u \in L_{\text {comp }}^{\infty}(\mathbb{R})$, for some $R>0$ we have

$$
\int_{-R}^{R}\left(\left|u_{+}(x)\right|^{p}+\left|u_{-}(x)\right|^{p}\right) d x<\infty, \quad \forall p<\infty,
$$

and

$$
\left|u_{+}(x)\right|+\left|u_{-}(x)\right| \leq C|x|^{-1}, \quad|x|>R,
$$

and similar bounds hold for $v_{ \pm}$. Recall also that $f \in L^{p}(-R, R)$ for any $R>0$ and any $p<\infty$, and the integral (5.1) converges. Putting this together, we see that the integrals

$$
\int_{-\infty}^{\infty} f(x) u_{-}(x) \overline{v_{+}(x)} d x \quad \text { and } \quad \int_{-\infty}^{\infty} f(x) u_{+}(x) \overline{v_{-}(x)} d x
$$

converge absolutely, and so the inner products in (5.4) are well defined. Although these inner products need not make sense for arbitrary $u, v \in L^{2}$, below we will see that $(\breve{f} u, v)$ is bounded in $u, v$ in the $L^{2}$ norm, and therefore $\breve{f}$ extends as a bounded operator to $L^{2}$.

Further, we have

$$
\frac{1}{2 \pi i} \check{f}=P_{+} f P_{-}-P_{-} f P_{+}=\left(\begin{array}{cc}
0 & P_{+} f P_{-} \\
-P_{-} f P_{+} & 0
\end{array}\right)
$$

with respect to the orthogonal decomposition $L^{2}(\mathbb{R})=\operatorname{Ran} P_{+} \oplus \operatorname{Ran} P_{-}$. This gives an immediate (and well-known) connection with Hankel operators. For $f \in$ $\operatorname{BMO}(\mathbb{R})$, the Hankel operator $H(f)$ is defined by

$$
H(f): H^{2}\left(\mathbb{C}_{+}\right) \rightarrow H^{2}\left(\mathbb{C}_{-}\right), \quad H(f) u=P_{-}(f u), \quad u \in H^{2}\left(\mathbb{C}_{+}\right) .
$$

Thus, $P_{-} f P_{+}$is exactly the Hankel operator $H(f)$ but defined on the wider space $L^{2}(\mathbb{R})$; in particular, the operator norm (and all Schatten norms) of the operators $P_{-} f P_{+}$and $H(f)$ coincide. This shows that the required results on the boundedness 
and Schatten class properties of $\check{f}$ follow directly from the corresponding known results on Hankel operators. Below we make this explicit.

\subsection{Boundedness of $\breve{f}$.}

Lemma 5.2. (i) Let $f \in \mathrm{BMO}(\mathbb{R})$. Then the sesquilinear form (5.4) satisfies the bound

$$
|(\check{f} u, v)| \leq 2 \pi\|f\|_{\mathrm{BMO}}\|u\|_{L^{2}}\|v\|_{L^{2}}, \quad u, v \in L_{\mathrm{comp}}^{\infty}(\mathbb{R}) .
$$

Thus, $\check{f}$ extends to a bounded operator on $L^{2}(\mathbb{R})$. Further, one has

$$
\|\check{f}\|=2 \pi\|f\|_{\text {BMO }}
$$

(ii) If $f_{n} \rightarrow f *$-weakly in $\mathrm{BMO}$, then $\check{f}_{n} \rightarrow \check{f} *$-weakly in $\mathcal{B}\left(L^{2}(\mathbb{R})\right)$.

Proof. Let us first consider the quadratic form

$$
\left(f u_{+}, v_{-}\right)=\int_{-\infty}^{\infty} f(x) u_{+}(x) \overline{v_{-}(x)} d x
$$

for $u, v \in L_{\text {comp }}^{\infty}(\mathbb{R})$. As already discussed, the integral here converges absolutely. Further, since $u_{+}, \overline{v_{-}} \in H^{2}\left(\mathbb{C}_{+}\right)$, we have $u_{+} \overline{v_{-}} \in H^{1}\left(\mathbb{C}_{+}\right)$and so

$$
\left(f u_{+}, v_{-}\right)=T_{f}\left(u_{+} \overline{v_{-}}\right) \text {. }
$$

It follows that

$$
\left|\left(f u_{+}, v_{-}\right)\right|=\left|T_{f}\left(u_{+} \overline{v_{-}}\right)\right| \leq\left\|T_{f}\right\|_{H^{1}\left(\mathbb{C}_{+}\right)^{*}}\left\|u_{+} \overline{v_{-}}\right\|_{H^{1}\left(\mathbb{C}_{+}\right)} \leq\left\|T_{f}\right\|_{H^{1}\left(\mathbb{C}_{+}\right)^{*}}\|u\|_{L^{2}}\|v\|_{L^{2}},
$$

which can be written as

$$
\left\|P_{-} f P_{+}\right\| \leq\left\|T_{f}\right\|_{H^{1}\left(\mathbb{C}_{+}\right)^{*}}
$$

Further, since (see e.g. [7, Exercise II.1]) any function in $H^{1}\left(\mathbb{C}_{+}\right)$can be represented as $u_{+} \overline{v_{-}}$with

$$
\left\|u_{+} \overline{v_{-}}\right\|_{H^{1}\left(\mathbb{C}_{+}\right)}=\left\|u_{+}\right\|_{L^{2}}\left\|v_{-}\right\|_{L^{2}}
$$

it is easy to see that in fact we have the equality of the norms,

$$
\left\|P_{-} f P_{+}\right\|=\left\|T_{f}\right\|_{H^{1}\left(\mathbb{C}_{+}\right)^{*}} .
$$

Similarly,

$$
\left(f u_{-}, v_{+}\right)=\overline{T_{\bar{f}}\left(v_{+} \overline{u_{-}}\right)} \text {and }\left\|P_{+} f P_{-}\right\|=\left\|T_{\bar{f}}\right\|_{H^{1}\left(\mathbb{C}_{+}\right)^{*}} .
$$

Now by (5.5) we obtain

$\frac{1}{2 \pi}\|\check{f}\|=\max \left\{\left\|P_{-} f P_{+}\right\|,\left\|P_{+} f P_{-}\right\|\right\}=\max \left\{\left\|T_{f}\right\|_{H^{1}\left(\mathbb{C}_{+}\right)^{*}},\left\|T_{\bar{f}}\right\|_{H^{1}\left(\mathbb{C}_{+}\right)^{*}}\right\}=\|f\|_{\mathrm{BMO}}$, according to our definition of the BMO norm.

This argument also shows that if $f_{n} \rightarrow 0 *$-weakly in BMO, then

$$
\left(f_{n} u_{+}, v_{-}\right)=T_{f_{n}}\left(u_{+} \overline{v_{-}}\right) \rightarrow 0
$$


and

which yields (ii).

$$
\left(f_{n} u_{-}, v_{+}\right)=\overline{T_{\overline{f_{n}}}\left(v_{+} \overline{u_{-}}\right)} \rightarrow 0
$$

Lemma 5.3. If $f \in \mathcal{R}$, then the operator $\check{f}$ has a finite rank. If $f \in \operatorname{CMO}(\mathbb{R})$, then the operator $\breve{f}$ is compact.

Proof. Let $f(x)=\left(x-z_{0}\right)^{-1}, \operatorname{Im} z_{0} \neq 0$. Then

$$
\check{f}(x, y)=\frac{\left(x-z_{0}\right)^{-1}-\left(y-z_{0}\right)^{-1}}{x-y}=-\left(x-z_{0}\right)^{-1}\left(y-z_{0}\right)^{-1}
$$

so $\check{f}$ is a rank one operator. Differentiating (5.7) $m$ times with respect to $z_{0}$, one checks that $\check{f}$ is finite rank for $f(x)=\left(x-z_{0}\right)^{-m-1}$. By partial fraction decomposition, we get that $\breve{f}$ is finite rank for any rational $f$.

Now let $f \in \mathrm{CMO}(\mathbb{R})$; approximating $f$ by rational functions in BMO norm, we obtain in view of (15.6) an approximation of $\breve{f}$ by finite rank operators in the operator norm. Thus, $\check{f}$ is compact.

5.6. Schatten class properties of $\breve{f}$. Below we state Peller's characterisation of Hankel operators of Schatten class in a form convenient for us. For the proofs and the history, see [14, Chapter 6].

Proposition 5.4. [14, Theorem 6.7.4] For any $0<p<\infty$, there exist constants $c_{1}(p)<C_{1}(p)$ such that for all $f \in B_{p, p}^{1 / p}(\mathbb{R}) \cap \operatorname{BMO}(\mathbb{R})$,

$$
c_{1}(p)^{p}\|f\|_{B_{p, p}^{1 / p}}^{p} \leq\|H(f)\|_{p}^{p}+\|H(\bar{f})\|_{p}^{p} \leq C_{1}(p)^{p}\|f\|_{B_{p, p}^{1 / p}}^{p} .
$$

Remark. Of course, the constants $C_{1}(p)$ and $c_{1}(p)$ depend on the choice of the functional $\|\cdot\|_{B_{p, p}^{1 / p}}$ in $B_{p, p}^{1 / p}(\mathbb{R})$. The bounds (5.8) are not explicitly stated in [14, Theorem 6.7.4], but are obtained in the proof of that theorem.

Lemma 5.5. For any $0<p<\infty$, one has

$$
(2 \pi)^{-p}\|\breve{f}\|_{p}^{p}=\|H(f)\|_{p}^{p}+\|H(\bar{f})\|_{p}^{p}, \quad f \in B_{p, p}^{1 / p}(\mathbb{R}) .
$$

Thus, we have the estimates

$$
c_{1}(p)\|f\|_{B_{p, p}^{1 / p}} \leq(2 \pi)^{-1}\|\check{f}\|_{p} \leq C_{1}(p)\|f\|_{B_{p, p}^{1 / p},}, \quad f \in B_{p, p}^{1 / p}(\mathbb{R}),
$$

with the constants as in Proposition 5.4.

Proof. By (5.5), we have

$$
\frac{1}{2 \pi i} \check{f}=\left(\begin{array}{cc}
0 & \left(P_{-} \bar{f} P_{+}\right)^{*} \\
-P_{-} f P_{+} & 0
\end{array}\right) \text { in } L^{2}(\mathbb{R})=\operatorname{Ran} P_{+} \oplus \operatorname{Ran} P_{-} .
$$

Now the required result follows from the fact that (by a simple calculation)

$$
\|X\|_{p}^{p}=\|A\|_{p}^{p}+\|B\|_{p}^{p} \quad \text { for } X=\left(\begin{array}{cc}
0 & A \\
B & 0
\end{array}\right) .
$$




\section{The MAP $f \mapsto D(f)$}

6.1. Overview. In this section we put together all the components prepared so far. Throughout this section, $H_{0}$ and $H_{1}$ are self-adjoint operators in a Hilbert space $\mathcal{H}$ and $G_{0}, G_{1}$ are linear operators from $\mathcal{H}$ to $\mathcal{K}$ such that

$$
G_{0} \in \operatorname{Smooth}\left(H_{0}\right) \quad \text { and } \quad G_{1} \in \operatorname{Smooth}\left(H_{1}\right) .
$$

We assume that

$$
H_{1}-H_{0}=G_{1}^{*} G_{0}
$$

in the sense to be made precise later. We consider the map $f \mapsto D(f)$ in an abstract fashion, as a linear map from some function spaces to some spaces of operators. Our aim is to prove Theorems 1.1 and 1.2, which are restated more precisely as Theorems 6.2 and 6.5 below. The key step is the use of the Birman-Solomyak formula (1.8), which allows us to use the results of Sections 4 and 5 ,

6.2. Preliminaries. First we should explain that the identity (6.2) will be understood in the sesquilinear form sense:

$$
\left(u, H_{1} v\right)-\left(H_{0} u, v\right)=\left(G_{0} u, G_{1} v\right), \quad u \in \operatorname{Dom}\left(H_{0}\right), \quad v \in \operatorname{Dom}\left(H_{1}\right) .
$$

Next, since functions $f \in \mathrm{BMO}(\mathbb{R})$ need not be bounded, the operators $f\left(H_{0}\right)$ and $f\left(H_{1}\right)$ are in general unbounded for such $f$. Thus, the definition of $D(f)$ requires some care. Similarly to (6.3), we define the sesquilinear form of $D(f)$ as follows:

$$
d_{f}[u, v]:=\left(u, \bar{f}\left(H_{1}\right) v\right)-\left(f\left(H_{0}\right) u, v\right), \quad u \in \operatorname{Dom}\left(f\left(H_{0}\right)\right), \quad v \in \operatorname{Dom}\left(f\left(H_{1}\right)\right) .
$$

Obviously, for bounded functions $f$ one can define $D(f)$ directly as a bounded operator on $\mathcal{H}$ and in this case we have

$$
d_{f}[u, v]=(D(f) u, v), \quad u \in \operatorname{Dom} f\left(H_{0}\right), \quad v \in \operatorname{Dom} f\left(H_{1}\right) .
$$

In what follows we will prove that for any $f \in \operatorname{BMO}(\mathbb{R})$ the sesquilinear form $d_{f}[u, v]$ is bounded and therefore (6.5) holds with some bounded operator $D(f)$ in $\mathcal{H}$.

We denote

$$
R_{0}(z)=\left(H_{0}-z\right)^{-1}, \quad R_{1}(z)=\left(H_{1}-z\right)^{-1}, \quad \operatorname{Im} z \neq 0 .
$$

We will need the resolvent identity for operators satisfying (6.3); it can be written in two alternative forms:

$$
\begin{aligned}
& R_{1}(z)-R_{0}(z)=-\left(G_{1} R_{1}(\bar{z})\right)^{*} G_{0} R_{0}(z), \\
& R_{1}(z)-R_{0}(z)=-\left(G_{0} R_{0}(\bar{z})\right)^{*} G_{1} R_{1}(z),
\end{aligned}
$$

for any $\operatorname{Im} z \neq 0$.

First we give a simple statement reducing the analysis of $D(f)$ to the absolutely continuous subspaces of $H_{0}$ and $H_{1}$. 
Proposition 6.1. Assume (6.1) and (6.3). Then for the quadratic form $d_{f}$, defined by (6.5), we have $d_{f}[u, v]=0$ if $u \in \mathcal{H}^{(\text {sing })}\left(H_{0}\right) \cap \operatorname{Dom}\left(f\left(H_{0}\right)\right)$ or $v \in \mathcal{H}^{(\text {sing })}\left(H_{1}\right) \cap$ $\operatorname{Dom}\left(f\left(H_{1}\right)\right)$ (or both).

Proof. Suppose $u \in \mathcal{H}^{(\operatorname{sing})}\left(H_{0}\right)$; then for any $\operatorname{Im} z \neq 0$ we have $G R_{0}(z) u=0$ and therefore, by the resolvent identity (6.6),

$$
R_{1}(z) u=R_{0}(z) u \text {. }
$$

By Stone's formula [17, Theorem VII.13], this implies that the corresponding two spectral measures coincide on $u$ :

$$
E_{H_{0}}(\Delta) u=E_{H_{1}}(\Delta) u, \quad \forall \Delta \subset \mathbb{R} .
$$

It follows that

$$
\left(f\left(H_{0}\right) u, v\right)=\left(u, \bar{f}\left(H_{1}\right) v\right)
$$

whenever both sides are well-defined, i.e. whenever $u \in \operatorname{Dom} f\left(H_{0}\right)$ and $v \in$ $\operatorname{Dom} f\left(H_{1}\right)$. This is the equality $d_{f}[u, v]=0$ written in a different form.

The case $v \in \mathcal{H}^{(\text {sing })}\left(H_{1}\right)$ is considered in the same way, by using the resolvent identity in the form (6.7).

The above proposition is well known in scattering theory as the statement that under the assumptions (6.1), (6.2), the singular parts of $H_{0}$ and $H_{1}$ coincide. As a consequence of this proposition, when dealing with the sesquilinear from $d_{f}[u, v]$, it suffices to consider $u \in \mathcal{H}^{(\mathrm{ac})}\left(H_{0}\right)$ and $v \in \mathcal{H}^{(\mathrm{ac})}\left(H_{1}\right)$. In fact, the argument of Proposition 6.1 also shows that these absolutely continuous subspaces coincide: $\mathcal{H}^{(\mathrm{ac})}\left(H_{0}\right)=\mathcal{H}^{(\mathrm{ac})}\left(H_{1}\right)$, although we will not need this.

\subsection{The norm bound for $D(f)$.}

Theorem 6.2. For any $f \in \operatorname{BMO}(\mathbb{R})$ and for dense sets of $u \in \mathcal{H}^{(a c)}\left(H_{0}\right), v \in$ $\mathcal{H}^{(a c)}\left(H_{1}\right)$, the form (6.4) satisfies the bound

$$
\left|d_{f}[u, v]\right| \leq 2 \pi A\|f\|_{\mathrm{BMO}}\|u\|_{\mathcal{H}}\|v\|_{\mathcal{H}},
$$

where $A$ is the constant (4.5). Thus, the form $d_{f}[u, v]$ corresponds to a bounded operator $D(f)$ on $\mathcal{H}$ in the sense of (6.5), and $D(f)$ satisfies the norm bound

$$
\|D(f)\|_{\mathcal{B}} \leq 2 \pi A\|f\|_{\mathrm{BMO}(\mathbb{R})} .
$$

If $f_{n} \rightarrow f *$-weakly in $\mathrm{BMO}(\mathbb{R})$, then $D\left(f_{n}\right) \rightarrow D(f) *$-weakly in $\mathcal{B}(\mathcal{H})$.

Proof. We will prove the bound (6.8) for all $u \in L_{\text {comp }}^{\infty}\left(H_{1}\right), v \in L_{\text {comp }}^{\infty}\left(H_{0}\right)$ (see Section 2.1 for the definition of $\left.L_{\text {comp }}^{\infty}(H)\right)$. Since $L_{\text {comp }}^{\infty}\left(H_{j}\right)$ is dense in $\mathcal{H}^{(\text {ac) }}\left(H_{j}\right)$, $j=0,1$, this will suffice.

Since $v \in L_{\text {comp }}^{\infty}\left(H_{1}\right)$, the measure $\left(E_{H_{1}}(\cdot) u, v\right)$ is absolutely continuous and the function

$$
a(\lambda):=\frac{d\left(E_{H_{1}}(\lambda) u, v\right)}{d \lambda}
$$


is in $L_{\text {comp }}^{2}(\mathbb{R})$. It follows that (here $P_{\varepsilon}$ is the Poisson kernel (2.6) )

$$
\begin{aligned}
\left(u, \bar{f}\left(H_{1}\right) v\right)=\int_{-\infty}^{\infty} f(x) a(x) d x=\lim _{\varepsilon \rightarrow 0+} \int_{-\infty}^{\infty} f(x)\left(P_{\varepsilon} * a\right)(x) d x \\
=\frac{1}{2 \pi i} \lim _{\varepsilon \rightarrow 0+} \int_{-\infty}^{\infty} f(x)\left(\left(R_{1}(x+i \varepsilon)-R_{1}(x-i \varepsilon)\right) u, v\right) d x
\end{aligned}
$$

Similarly, we obtain

$$
\left(f\left(H_{0}\right) u, v\right)=\frac{1}{2 \pi i} \lim _{\varepsilon \rightarrow 0+} \int_{-\infty}^{\infty} f(x)\left(\left(R_{0}(x+i \varepsilon)-R_{0}(x-i \varepsilon)\right) u, v\right) d x .
$$

Let us subtract the last two identities one from another and use the resolvent identity (6.6). Denoting

$$
F_{u, v}(z)=\left(G_{0} R_{0}(z) u, G_{1} R_{1}(\bar{z}) v\right), \quad F_{u, v}^{*}(z)=\overline{F_{u, v}(\bar{z})}, \quad \operatorname{Im} z \neq 0
$$

we obtain

$$
\begin{aligned}
d_{f}[u, v]=-\frac{1}{2 \pi i} \lim _{\varepsilon \rightarrow 0+} \int_{-\infty}^{\infty} & f(x)\left(F_{u, v}(x+i \varepsilon)-F_{u, v}(x-i \varepsilon)\right) d x \\
= & -\frac{1}{2 \pi i} \lim _{\varepsilon \rightarrow 0+} \int_{-\infty}^{\infty} f(x)\left(F_{u, v}(x+i \varepsilon)-\overline{F_{u, v}^{*}(x+i \varepsilon)}\right) d x .
\end{aligned}
$$

By the definition (2.1) of Kato smoothness, the functions $F_{u, v}$ and $F_{u, v}^{*}$ belong to $H^{1}\left(\mathbb{C}_{+}\right)$. Thus, in notation (5.2) the previous identity can be written as

$$
d_{f}[u, v]=-\frac{1}{2 \pi i}\left(T_{f}\left(F_{u, v}\right)-\overline{T_{\bar{f}}\left(F_{u, v}^{*}\right)}\right) .
$$

We have

$$
\begin{aligned}
& \int_{-\infty}^{\infty}\left|F_{u, v}(x+i \varepsilon)\right| d x \leq \int_{-\infty}^{\infty}\left\|G_{0} R_{0}(x+i \varepsilon) u\right\|\left\|G_{1} R_{1}(x-i \varepsilon) v\right\| d x \\
& \leq \frac{\alpha}{2} \int_{-\infty}^{\infty}\left\|G_{0} R_{0}(x+i \varepsilon) u\right\|^{2} d x+\frac{1}{2 \alpha} \int_{-\infty}^{\infty}\left\|G_{1} R_{1}(x-i \varepsilon) v\right\|^{2} d x
\end{aligned}
$$

where $\alpha>0$ is a parameter to be chosen later. Similiarly,

$$
\int_{-\infty}^{\infty}\left|F_{u, v}^{*}(x+i \varepsilon)\right| d x \leq \frac{\alpha}{2} \int_{-\infty}^{\infty}\left\|G_{0} R_{0}(x-i \varepsilon) u\right\|^{2} d x+\frac{1}{2 \alpha} \int_{-\infty}^{\infty}\left\|G_{1} R_{1}(x+i \varepsilon) v\right\|^{2} d x .
$$

By the definition (2.1) of Kato smoothness, we get

$$
\begin{aligned}
\int_{-\infty}^{\infty}\left(\left|F_{u, v}(x+i \varepsilon)\right|\right. & \left.+\left|F_{u, v}^{*}(x+i \varepsilon)\right|\right) d x \\
& \leq \frac{\alpha}{2}(2 \pi)^{2}\left\|G_{0}\right\|_{\operatorname{Smooth}\left(H_{0}\right)}^{2}\|u\|^{2}+\frac{1}{2 \alpha}(2 \pi)^{2}\left\|G_{1}\right\|_{\operatorname{Smooth}\left(H_{1}\right)}^{2}\|v\|^{2} .
\end{aligned}
$$


Optimising over $\alpha$, we obtain

$$
\int_{-\infty}^{\infty}\left(\left|F_{u, v}(x+i \varepsilon)\right|+\left|F_{u, v}^{*}(x+i \varepsilon)\right|\right) d x \leq(2 \pi)^{2} A\|u\|\|v\| .
$$

Now coming back to (6.9), we have

$$
\begin{aligned}
& 2 \pi\left|d_{f}[u, v]\right| \leq\left\|T_{f}\right\|_{H^{1}\left(\mathbb{C}_{+}\right)^{*}}\left\|F_{u, v}\right\|_{H^{1}\left(\mathbb{C}_{+}\right)}+\left\|T_{\bar{f}}\right\|_{H^{1}\left(\mathbb{C}_{+}\right)^{*}}\left\|F_{u, v}^{*}\right\|_{H^{1}\left(\mathbb{C}_{+}\right)} \\
& \leq\|f\|_{\mathrm{BMO}}\left(\left\|F_{u, v}\right\|_{H^{1}\left(\mathbb{C}_{+}\right)}+\left\|F_{u, v}^{*}\right\|_{H^{1}\left(\mathbb{C}_{+}\right)}\right) \leq(2 \pi)^{2}\|f\|_{\mathrm{BMO}} A\|u\|\|v\|,
\end{aligned}
$$

as required.

Finally, suppose $f_{n} \rightarrow f *$-weakly in BMO. Consider the identity (6.9). It has been proven above for $u \in L_{\text {comp }}^{\infty}\left(H_{0}\right), v \in L_{\text {comp }}^{\infty}\left(H_{1}\right)$; but since we already know that $d_{f}[u, v]$ is bounded, it extends by a limiting argument to all $u, v \in \mathcal{H}$. By the definition of $*$-weak convergence in $\mathrm{BMO}(\mathbb{R})$ we deduce from $(6.9)$ that

$$
\left(D\left(f_{n}\right) u, v\right) \rightarrow(D(f) u, v), \quad u, v \in \mathcal{H}
$$

as required.

6.4. Birman-Solomyak formula. Here we discuss the Birman-Solomyak formula (1.8). As in Section 4, we use the shorthand notation DOI $(a)$, see (4.1). In our framework, the Birman-Solomyak formula becomes

Theorem 6.3. For all $f \in \mathrm{BMO}(\mathbb{R})$, the identity

$$
D(f)=\operatorname{DOI}(\check{f})
$$

holds true.

Proof. First let us check (6.10) for $f(x)=\left(x-z_{0}\right)^{-1}, \operatorname{Im} z_{0} \neq 0$. By the resolvent identity (6.6), we have

$$
\begin{gathered}
D(f)=R_{1}\left(z_{0}\right)-R_{0}\left(z_{0}\right)=-\left(G_{1} R_{1}\left(\overline{z_{0}}\right)\right)^{*} G_{0} R_{0}\left(z_{0}\right), \\
\check{f}(x, y)=\frac{\left(x-z_{0}\right)^{-1}-\left(y-z_{0}\right)^{-1}}{x-y}=-\left(x-z_{0}\right)^{-1}\left(y-z_{0}\right)^{-1},
\end{gathered}
$$

and so, by the definition (4.7) of DOI,

$$
\operatorname{DOI}(\check{f})=-\left(G_{1}\left(H_{1}-\overline{z_{0}}\right)^{-1}\right)^{*} G_{0}\left(H_{0}-z_{0}\right)^{-1}=D(f),
$$

as claimed. Next, if $f(x)=\left(x-z_{0}\right)^{-1-m}, m \geq 0$, then the required identity follows by differentiating $m$ times with respect to $z_{0}$. By partial fraction decomposition, it follows that (6.10) holds true for all $f \in \mathcal{R}$.

Now let us extend (6.10) to all $f \in \operatorname{BMO}(\mathbb{R})$ by using $*$-weak convergence. Rational functions are $*$-weak dense in BMO by Lemma 5.1, The left side of (6.10) is continuous with respect to $*$-weak convergence by Theorem 6.2, The map $f \mapsto \check{f}$ is *-weak continuous by Lemma $5.2($ ii $)$, and the map $\breve{f} \mapsto \operatorname{DOI}(\check{f})$ is *-weak continuous by Lemma 4.3 (and because we have defined DOI to be the 
*-weak continuous extension from finite rank operators). Thus, (6.10) holds true for all $f \in \operatorname{BMO}(\mathbb{R})$.

\subsection{Compactness and Schatten class properties of $D(f)$.}

Theorem 6.4. Let $f \in \operatorname{CMO}(\mathbb{R})$ and $G_{0} \in \operatorname{Smooth}\left(H_{0}\right), G_{1} \in \operatorname{Smooth}\left(H_{1}\right)$. Assume in addition that either $G_{0} \in \operatorname{Smooth}_{\infty}\left(H_{0}\right)$ or $G_{1} \in \operatorname{Smooth}_{\infty}\left(H_{1}\right)$. Then $D(f)$ is compact.

Proof. By Theorem [6.3, it suffices to check that DOI $(\check{f})$ is compact. Here $\breve{f}$ is compact by Lemma 5.3. Now the result follows from Lemma 4.5.

Finally, we can prove our main result for Schatten classes, which is Theorem 1.2 . We state it again for convenience:

Theorem 6.5. Let $p, q, r$ be finite positive indices satisfying $\frac{1}{p}=\frac{1}{q}+\frac{1}{r}$. Let $G_{0} \in \operatorname{Smooth}_{q}\left(H_{0}\right)$ and $G_{1} \in \operatorname{Smooth}_{r}\left(H_{1}\right)$. Then for any $f \in B_{p, p}^{1 / p}(\mathbb{R}) \cap \operatorname{BMO}(\mathbb{R})$, we have $D(f) \in \mathbf{S}_{p}$ and

$$
\|D(f)\|_{p} \leq(2 \pi) C_{1}(p) A_{q, r}\|f\|_{B_{p, p}^{1 / p}(\mathbb{R})},
$$

where $C_{1}(p)$ is the constant from (5.8) and $A_{q, r}$ is the constant from (4.5). This extends to $q=\infty$ (resp. $r=\infty)$, if the class $\operatorname{Smooth}_{q}\left(H_{0}\right)$ (resp. $\left.\operatorname{Smooth}_{r}\left(H_{1}\right)\right)$ is replaced by $\operatorname{Smooth}\left(H_{0}\right)$ (resp. $\operatorname{Smooth}\left(H_{1}\right)$ ).

Proof. By Theorem 6.3, Theorem 4.6 and Lemma 5.5, we have

$$
\|D(f)\|_{p}=\|\operatorname{DOI}(\check{f})\|_{p} \leq A_{q, r}\|\check{f}\|_{p} \leq A_{q, r}(2 \pi) C_{1}(p)\|f\|_{B_{p, p}^{1 / p}(\mathbb{R})} .
$$

\section{SHARPNESS AND SOME EXTENSIONS}

This section contains some additional information. We demonstrate the sharpness of our main result and give some extensions.

7.1. Sharpness of estimates. Here we construct a pair of self-adjoint operators $H_{0}, H_{1}$ in $L^{2}(\mathbb{R})$ such that the estimates from Theorems 1.1 and 1.2 are saturated. Thus, this construction demonstrates that these estimates are sharp. We construct $H_{0}$ and $H_{1}$ as follows.

Let $H_{0}$ be the multiplication operator $\mathcal{M}$ in $L^{2}(\mathbb{R})$ from (2.9). Let $J$ be the Hilbert transform,

$$
J f(x)=\frac{1}{\pi i} \text { p.v. } \int_{-\infty}^{\infty} \frac{f(y)}{y-x} d y, \quad f \in L^{2}(\mathbb{R}) .
$$

It is well known that $J$ is unitary in $L^{2}(\mathbb{R})$; it is also evident that $J^{*}=J$. We set

$$
H_{1}=J H_{0} J, \quad \operatorname{Dom} H_{1}=\left\{J u: u \in \operatorname{Dom} H_{0}\right\} .
$$


Next, we would like to represent the difference $H_{1}-H_{0}$ as a product $G_{1}^{*} G_{0}$. Let $\mathcal{K}=\mathbb{C}$ and let $G_{0}: L^{2}(\mathbb{R}) \rightarrow \mathbb{C}$ be as in Example 2.5.

$$
G_{0} u=\int_{-\infty}^{\infty} u(x) d x, \quad u \in \operatorname{Dom} H_{0}
$$

The operator $G_{0}$ is not closable, but $G_{0} \in \operatorname{Smooth}\left(H_{0}\right)$, with $\left\|G_{0}\right\|_{\operatorname{Smooth}\left(H_{0}\right)}=1$. Further, we set

$$
G_{1} u=\frac{1}{\pi i} G_{0} J u, \quad \operatorname{Dom} G_{1}=\operatorname{Dom} H_{1} .
$$

Clearly, $G_{1} \in \operatorname{Smooth}\left(H_{1}\right)$ with $\left\|G_{1}\right\|_{\operatorname{Smooth}\left(H_{1}\right)}=1 / \pi$. Thus, the constant $A$ (see (4.5)) equals $A=1 / \pi$ in this case. We have

Theorem 7.1. Let $H_{0}, H_{1}, G_{0}, G_{1}$ be as described above. Then:

(i) The identity (6.3) holds true (i.e. $H_{1}=H_{0}+G_{1}^{*} G_{0}$ in the sesquilinear form sense).

(ii) For any $f \in \operatorname{BMO}(\mathbb{R})$, we have

Thus,

$$
f\left(H_{1}\right)-f\left(H_{0}\right)=\frac{1}{\pi i} \check{f} J
$$

$$
\begin{gathered}
\left\|f\left(H_{1}\right)-f\left(H_{0}\right)\right\|=\frac{1}{\pi}\|\breve{f}\|=2\|f\|_{\mathrm{BMO}}=(2 \pi) A\|f\|_{\mathrm{BMO}} \\
f \in B_{p, p}^{1 / p}(\mathbb{R}) \cap \operatorname{BMO}(\mathbb{R}) \Leftrightarrow D(f) \in \mathbf{S}_{p} .
\end{gathered}
$$

Proof. Let $u, w \in L_{\text {comp }}^{\infty}(\mathbb{R})$, and let $v=J w$. Consider the left side of (6.3):

$$
\begin{aligned}
\left(u, H_{1} v\right)-\left(H_{0} u, v\right) & =\left(u, J H_{0} w\right)-\left(H_{0} u, J w\right)=\left(J u, H_{0} w\right)-\left(J H_{0} u, w\right) \\
= & \frac{1}{\pi i} \int_{\mathbb{R}} \int_{\mathbb{R}} \frac{u(y)(x-y) \overline{w(x)}}{y-x} d y d x=-\frac{1}{\pi i} \overline{G_{0} w} G_{0} u=\overline{G_{1} v} G_{0} u,
\end{aligned}
$$

which is the right side of $(\underline{6.3})$. Next,

$$
f\left(H_{1}\right)-f\left(H_{0}\right)=J f\left(H_{0}\right) J-f\left(H_{0}\right)=\left(J f\left(H_{0}\right)-f\left(H_{0}\right) J\right) J=\frac{1}{\pi i} \check{f} J .
$$

From here we get the first identity in (7.1). The middle identity in (7.1) follows from Lemma 5.2, and the rest follows from Lemma 5.5.

7.2. Quasicommutators. Let $H_{0}$ and $H_{1}$ be self-adjoint operators in $\mathcal{H}$, and let $J$ be a bounded operator in $\mathcal{H}$. Here we consider the so-called quasicommutators

$$
D_{J}(f):=f\left(H_{1}\right) J-J f\left(H_{0}\right) .
$$

Let us assume that

$$
H_{1} J-J H_{0}=G_{1}^{*} G_{0}
$$

with some operators $G_{0}, G_{1}$ acting from $\mathcal{H}$ to $\mathcal{K}$ such that

$$
G_{0} \in \operatorname{Smooth}\left(H_{0}\right) \quad \text { and } \quad G_{1} \in \operatorname{Smooth}\left(H_{1}\right) .
$$


As usual, (7.3) should be understood in the sesquilinear form sense, i.e.

$$
\left(J u, H_{1} v\right)-\left(H_{0} u, J^{*} v\right)=\left(G_{0} u, G_{1} v\right), \quad u \in \operatorname{Dom}\left(H_{0}\right), \quad v \in \operatorname{Dom}\left(H_{1}\right) .
$$

The resolvent identity in this case takes the form

$$
R_{1}(z) J-J R_{0}(z)=-\left(G_{1} R_{1}(\bar{z})\right)^{*} G_{0} R_{0}(z)=-\left(G_{0} R_{0}(\bar{z})\right)^{*} G_{1} R_{1}(z) .
$$

Similarly to (6.4), we define the sesquilinear form

$$
d_{J, f}[u, v]:=\left(J u, \bar{f}\left(H_{1}\right) v\right)-\left(f\left(H_{0}\right) u, J^{*} v\right), \quad u \in \operatorname{Dom} f\left(H_{0}\right), \quad v \in \operatorname{Dom} f\left(H_{1}\right) .
$$

For bounded functions $f$ the quasicommutator $D_{J}(f)$ can be defined directly as in (7.2) and

$$
d_{J, f}[u, v]=\left(D_{J}(f) u, v\right), \quad u \in \operatorname{Dom} f\left(H_{0}\right), \quad v \in \operatorname{Dom} f\left(H_{1}\right) .
$$

Similarly to Proposition 6.1, we have

Proposition 7.2. Assume (7.4) and (7.5). Then we have $d_{J, f}[u, v]=0$ if $u \in$ $\mathcal{H}^{(\text {sing })}\left(H_{0}\right) \cap \operatorname{Dom}\left(f\left(H_{0}\right)\right)$ or $v \in \mathcal{H}^{(\text {sing })}\left(H_{1}\right) \cap \operatorname{Dom}\left(f\left(H_{1}\right)\right)$ (or both).

Proof. If $u \in \mathcal{H}^{(\text {sing) }}\left(H_{0}\right)$, then for all $\operatorname{Im} z \neq 0$ we have $G_{0} R_{0}(z) u=0$ and so, by the resolvent identity (7.6),

$$
R_{1}(z) J u=J R_{0}(z) u
$$

From here, as in the proof of Proposition 6.1, we obtain $d_{J, f}[u, v]=0$ for any $f$ such that $u \in \operatorname{Dom} f\left(H_{0}\right)$ and $v \in \operatorname{Dom} f\left(H_{1}\right)$. The case $v \in \mathcal{H}^{(\text {sing })}\left(H_{1}\right)$ is considered in the same way.

In full analogy with Theorem 6.2, we have

Theorem 7.3. Assume (7.4) and (7.5). For any $f \in \operatorname{BMO}(\mathbb{R})$ and for all $u \in$ $\mathcal{H}^{(a c)}\left(H_{0}\right), v \in \mathcal{H}^{(a c)}\left(H_{1}\right)$, the sesquilinear form $d_{J, f}$ satisfies the bound

$$
\left|d_{J, f}[u, v]\right| \leq 2 \pi A\|f\|_{\mathrm{BMO}}\|u\|_{\mathcal{H}}\|v\|_{\mathcal{H}},
$$

where $A$ is the constant (4.5). Thus, the form $d_{J, f}[u, v]$ corresponds to a bounded operator $D_{J}(f)$ on $\mathcal{H}$ in the sense of (17.7), and $D_{J}(f)$ satisfies the norm bound

$$
\left\|D_{J}(f)\right\| \leq 2 \pi A\|f\|_{\mathrm{BMO}(\mathbb{R})} \text {. }
$$

If $f_{n} \rightarrow f *$-weakly in $\mathrm{BMO}(\mathbb{R})$, then $D_{J}\left(f_{n}\right) \rightarrow D_{J}(f) *$-weakly in $\mathcal{B}(\mathcal{H})$.

The proof repeats the proof of Theorem 6.2 word for word; the only difference is that the required resolvent identity in this case has the form (7.6).

Furthermore, repeating word for word the proof of Theorem 6.3, we establish the modified Birman-Solomyak formula

$$
D_{J}(f)=\operatorname{DOI}(\check{f})
$$

for all $f \in \operatorname{BMO}(\mathbb{R})$. Thus, we can apply the compactness Lemma 4.5 and the Schatten bounds Theorem 4.6: 
Theorem 7.4. Assume (7.4) and (7.5); let $D_{J}(f)$ be as defined above. Assume $f \in \mathrm{CMO}(\mathbb{R})$ and assume in addition that at least one of the inclusions

$$
G_{0} \in \operatorname{Smooth}_{\infty}\left(H_{0}\right), \quad G_{1} \in \operatorname{Smooth}_{\infty}\left(H_{1}\right)
$$

holds true. Then $D_{J}(f)$ is compact. Further, let $p, q, r$ be finite positive indices satisfying $\frac{1}{p}=\frac{1}{q}+\frac{1}{r}$, and let $A_{q, r}$ be as in (4.5). Then the Schatten class bound

$$
\left\|D_{J}(f)\right\|_{p} \leq 2 \pi C_{1}(p) A_{q, r}\|f\|_{B_{p, p}^{1 / p}(\mathbb{R})}
$$

holds true for all $f \in B_{p, p}^{1 / p}(\mathbb{R}) \cap \operatorname{BMO}(\mathbb{R})$. It extends to $q=\infty$ (resp. $\left.r=\infty\right)$, if one replaces the class $\operatorname{Smooth}_{q}\left(H_{0}\right)$ (resp. $\operatorname{Smooth}_{r}\left(H_{1}\right)$ ) by $\operatorname{Smooth}\left(H_{0}\right)$ (resp. $\left.\operatorname{Smooth}\left(H_{1}\right)\right)$.

7.3. Products of functions. Let $H_{0}$ and $H_{1}$ be self-adjoint operators in $\mathcal{H}$, and let $\varphi_{0}, \varphi_{1} \in L^{\infty}(\mathbb{R})$. Here we consider the products

$$
\varphi_{1}\left(H_{1}\right)^{*} D(f) \varphi_{0}\left(H_{0}\right),
$$

where $D(f)=f\left(H_{1}\right)-f\left(H_{0}\right)$ as before. The main interest of this is in taking $\varphi_{0}=\varphi_{1}=\mathbb{1}_{\Lambda}$, where $\Lambda \subset \mathbb{R}$; this leads to local variants of smoothness conditions. We develop this in more detail in the forthcoming publication [6].

We assume that

$$
H_{1}-H_{0}=G_{1}^{*} G_{0}
$$

for some $G_{0}, G_{1}: \mathcal{H} \rightarrow \mathcal{K}$, where $G_{0}$ is $H_{0}$-bounded and $G_{1}$ is $H_{1}$-bounded. As usual, (7.9) should be understood in the sesquilinear form sense, see (6.3). Our smoothness assumptions are now as follows:

$$
G_{0} \varphi_{0}\left(H_{0}\right) \in \operatorname{Smooth}\left(H_{0}\right), \quad G_{1} \varphi_{1}\left(H_{1}\right) \in \operatorname{Smooth}\left(H_{1}\right) .
$$

We define the operator (7.8) via the sesquilinear form

$$
d[u, v]:=\left(\varphi_{0}\left(H_{0}\right) u, \bar{f}\left(H_{1}\right) \varphi_{1}\left(H_{1}\right) v\right)-\left(f\left(H_{0}\right) \varphi_{0}\left(H_{0}\right) u, \varphi_{1}\left(H_{1}\right) v\right),
$$

for $u \in \operatorname{Dom} f\left(H_{0}\right)$ and $v \in \operatorname{Dom} f\left(H_{1}\right)$.

Theorem 7.5. Assume (17.9) and (7.10); let $f \in \mathrm{BMO}(\mathbb{R})$ and let $d$ be as above. Then $d[u, v]=0$, if $u \in \mathcal{H}^{(\text {sing })}\left(H_{0}\right) \cap \operatorname{Dom} f\left(H_{0}\right)$ or $v \in \mathcal{H}^{(\text {sing })\left(H_{1}\right)} \cap \operatorname{Dom} f\left(H_{1}\right)$. Further, for $u \in \mathcal{H}^{(a c)}\left(H_{0}\right)$ and $v \in \mathcal{H}^{(a c)}\left(H_{1}\right)$, the sesquilinear form d satisfies the bound

$$
|d[u, v]| \leq 2 \pi\|f\|_{\mathrm{BMO}(\mathbb{R})}\left\|G_{0} \varphi_{0}\left(H_{0}\right)\right\|_{\operatorname{Smooth}\left(H_{0}\right)}\left\|G_{1} \varphi_{1}\left(H_{1}\right)\right\|_{\operatorname{Smooth}\left(H_{1}\right)}\|u\|_{\mathcal{H}}\|v\|_{\mathcal{H}} .
$$

Thus, the sesquilinear form $d$ corresponds to a bounded operator $\varphi_{1}\left(H_{1}\right)^{*} D(f) \varphi_{0}\left(H_{0}\right)$, which satisfies

$\left\|\varphi_{1}\left(H_{1}\right)^{*} D(f) \varphi_{0}\left(H_{0}\right)\right\| \leq 2 \pi\|f\|_{\mathrm{BMO}(\mathbb{R})}\left\|G_{0} \varphi_{0}\left(H_{0}\right)\right\|_{\operatorname{Smooth}\left(H_{0}\right)}\left\|G_{1} \varphi_{1}\left(H_{1}\right)\right\|_{\operatorname{Smooth}\left(H_{1}\right)}$. If $f_{n} \rightarrow f *$-weakly in $\mathrm{BMO}(\mathbb{R})$, then

$$
\varphi_{1}\left(H_{1}\right)^{*} D\left(f_{n}\right) \varphi_{0}\left(H_{0}\right) \rightarrow \varphi_{1}\left(H_{1}\right)^{*} D(f) \varphi_{0}\left(H_{0}\right)
$$

*-weakly in $\mathcal{B}(\mathcal{H})$. 
Proof. Let

$$
J=\varphi_{1}\left(H_{1}\right)^{*} \varphi_{0}\left(H_{0}\right)
$$

and let $d_{J, f}$ be as defined in (7.7). Observe that we have

$$
H_{1} J-J H_{0}=\left(G_{1} \varphi_{1}\left(H_{1}\right)\right)^{*}\left(G_{0} \varphi_{0}\left(H_{0}\right)\right)
$$

in the sesquilinear form sense, and

$$
\varphi_{1}\left(H_{1}\right)^{*}\left(f\left(H_{1}\right)-f\left(H_{0}\right)\right) \varphi_{0}\left(H_{0}\right)=f\left(H_{1}\right) \varphi_{1}\left(H_{1}\right)^{*} \varphi_{0}\left(H_{0}\right)-\varphi_{1}\left(H_{1}\right)^{*} \varphi_{0}\left(H_{0}\right) f\left(H_{0}\right),
$$

or, in different notation,

$$
d[u, v]=d_{J, f}[u, v] .
$$

Thus, the operator identity

$$
\varphi_{1}\left(H_{1}\right)^{*} D(f) \varphi_{0}\left(H_{0}\right)=D_{J}(f)
$$

holds true and our claims follow immediately from Proposition 7.2 and Theorem 7.3

As an immediate consequence of (7.11) and of Theorem 7.4, we also obtain the corresponding compactness result and the Schatten norm bounds.

Theorem 7.6. Assume (7.9) and (7.10), and let $f \in \mathrm{CMO}(\mathbb{R})$. Assume that at least one of the two inclusions

$$
G_{0} \varphi_{0}\left(H_{0}\right) \in \operatorname{Smooth}_{\infty}\left(H_{0}\right), \quad G_{1} \varphi_{1}\left(H_{1}\right) \in \operatorname{Smooth}_{\infty}\left(H_{1}\right)
$$

holds true. Then $\varphi_{1}\left(H_{1}\right)^{*} D(f) \varphi_{0}\left(H_{0}\right)$ is compact. Further, let $p, q, r$ be finite positive indices such that $\frac{1}{p}=\frac{1}{q}+\frac{1}{r}$, and let $f \in B_{p, p}^{1 / p}(\mathbb{R}) \cap \operatorname{BMO}(\mathbb{R})$. Then we have the bounds

$$
\begin{aligned}
& \left\|\varphi_{1}\left(H_{1}\right)^{*} D(f) \varphi_{0}\left(H_{0}\right)\right\|_{p} \\
& \quad \leq 2 \pi C_{1}(p)\|f\|_{B_{p, p}^{1 / p}(\mathbb{R})}\left\|G_{0} \varphi_{0}\left(H_{0}\right)\right\|_{\operatorname{Smooth}_{q}\left(H_{0}\right)}\left\|G_{1} \varphi_{1}\left(H_{1}\right)\right\|_{\operatorname{Smooth}_{r}\left(H_{1}\right)} .
\end{aligned}
$$

This extends to the case $q=\infty$ (resp. $r=\infty$ ), if one replaces the class $\operatorname{Smooth}_{q}\left(H_{0}\right)\left(\right.$ resp. $\left.\operatorname{Smooth}_{r}\left(H_{1}\right)\right)$ by $\operatorname{Smooth}\left(H_{0}\right)$ (resp. $\left.\operatorname{Smooth}\left(H_{1}\right)\right)$.

\section{Appendix A. Two technical proofs}

Sketch of proof of Proposition 1.3. The key point is the calculation of the asymptotics of the Fourier transform of $F_{\alpha}$. A lengthy but straightforward calculation (see e.g. [16, Section 4]) yields that for $a_{+} \neq a_{-}$we have

$$
\widehat{F}_{\alpha}(t)=\frac{a_{+}-a_{-}}{2 \pi i} \frac{1}{t}(\log |t|)^{-\alpha}+O\left(t^{-1}(\log |t|)^{-\alpha-1}\right), \quad t \rightarrow \pm \infty,
$$

and for $a_{+}=a_{-}$we have

$$
\widehat{F}_{\alpha}(t)=a_{+} \alpha \frac{1}{t}(\log |t|)^{-\alpha-1}+O\left(t^{-1}(\log |t|)^{-\alpha-2}\right), \quad t \rightarrow \pm \infty .
$$


In both cases, the $O(\cdot)$ terms can be differentiated arbitrary many times, i.e.

$$
(d / d t)^{m} O\left(t^{-1}(\log |t|)^{-\alpha-1}\right)=O\left(t^{-1-m}(\log |t|)^{-\alpha-1}\right) .
$$

First consider the case $a_{+} \neq a_{-}$and let us check that the series (5.3) converges if and only if $\alpha>1 / p$. It is easy to see that $\widehat{F}_{\alpha}(t)$ is a $C^{\infty}$-smooth function of $t \in \mathbb{R}$ and as a consequence, the series over $j \leq 0$ converges for all $p>0$ and $\alpha \in \mathbb{R}$. Thus, it suffices to inspect the convergence of the series over $j \geq 0$. By the asymptotics (A.1), we have

$$
\begin{gathered}
\left(F_{\alpha} * \widehat{w}_{j}\right)(x)=\int_{0}^{\infty} e^{i x t} \widehat{F}_{\alpha}(t) w_{j}(t) d t=\frac{a_{+}-a_{-}}{2 \pi i} \int_{0}^{\infty} \frac{1}{t}(\log t)^{-\alpha} w\left(t / 2^{j}\right) e^{i x t} d t+\text { error } \\
=\frac{a_{+}-a_{-}}{2 \pi i} \int_{0}^{\infty} \frac{1}{t}\left(\log 2^{j} t\right)^{-\alpha} w(t) e^{i 2^{j} x t} d t+\text { error } \\
=\frac{a_{+}-a_{-}}{2 \pi i}\left(\log 2^{j}\right)^{-\alpha} \int_{0}^{\infty} \frac{1}{t}\left(1+\frac{\log t}{\log 2^{j}}\right)^{-\alpha} w(t) e^{i 2^{j} x t} d t+\text { error } \\
=\frac{a_{+}-a_{-}}{2 \pi i}\left(\log 2^{j}\right)^{-\alpha} \varphi\left(2^{j} x\right)+\text { error }
\end{gathered}
$$

where $\varphi$ is a Schwartz class function and the error term can be controlled by using the estimates (A.3). It follows that

$$
2^{j}\left\|F_{\alpha} * \widehat{w}_{j}\right\|_{L^{p}(\mathbb{R})}^{p}=C j^{-\alpha p}+o\left(j^{-\alpha p}\right), \quad j \rightarrow \infty,
$$

where $C \neq 0$. In the same way we get

$$
2^{j} \| F_{\alpha} *{\widehat{\widehat{w}_{j}}}_{L^{p}(\mathbb{R})}^{p}=C j^{-\alpha p}+o\left(j^{-\alpha p}\right), \quad j \rightarrow \infty .
$$

It follows that the series in (5.3) for $f=F_{\alpha}$ converges if and only if $p \alpha>1$.

In the same way, considering the case $a_{+}=a_{-}$and using the asymptotics (A.2), we conclude that the series (5.3) converges if and only if $p(\alpha+1)>1$.

Finally, we give the

Proof of Lemma 5.1. The proof is effected through mapping the problem to the unit circle.

Step 1: First we need to consider the analogous problem in the space $\mathrm{BMO}(\mathbb{T})$, which is defined as follows. For $h \in L^{2}(\mathbb{T})$ and $v \in H^{1}(\mathbb{D})$ (= the standard Hardy class on the unit disk), let

$$
t_{h}(v):=\lim _{r \rightarrow 1-} \int_{-\pi}^{\pi} h\left(e^{i \theta}\right) v\left(r e^{i \theta}\right) \frac{d \theta}{2 \pi},
$$

if the limit exists. Then $h \in \operatorname{BMO}(\mathbb{T})$ if and only if both linear functionals $t_{h}$ and $t_{\bar{h}}$ are bounded on $H^{1}(\mathbb{D})$. 
Let us prove that for $h \in \mathrm{BMO}(\mathbb{T})$, its approximations by Fejer sums converge to $h *$-weakly in BMO. More precisely, set

$$
h_{n}(z)=\sum_{j=-n}^{n}\left(1-\frac{|j|}{n}\right) \hat{h}_{j} z^{j}, \quad \text { where } \quad h(z)=\sum_{j=-\infty}^{\infty} \hat{h}_{j} z^{j} .
$$

It is easy to see that the linear map $h \mapsto h_{n}$ is bounded on $H^{1}(\mathbb{D})$ :

$$
\left\|h_{n}\right\|_{H^{1}(\mathbb{D})} \leq C\|h\|_{H^{1}(\mathbb{D})},
$$

and so, by duality,

$$
\left\|h_{n}\right\|_{\mathrm{BMO}(\mathbb{D})} \leq C\|h\|_{\mathrm{BMO}(\mathbb{D})} .
$$

Next, it is clear that if $v \in H^{1}(\mathbb{D})$ is a trigonometric polynomial, then

$$
t_{h_{n}}(v) \rightarrow t_{h}(v), \quad n \rightarrow \infty .
$$

Since trigonometric polynomials are dense in $H^{1}(\mathbb{D})$, by an approximation argument (involving (A.5) $)$, we obtain $\left(\right.$ A.6) for all $v \in H^{1}(\mathbb{D})$. Similarly, one proves that $t_{\bar{h}_{n}}(v) \rightarrow t_{\bar{h}}(v)$.

Step 2: Let $\omega$ be the standard conformal map from the unit disk to the upper half-plane:

$$
\omega: \mathbb{D} \rightarrow \mathbb{C}_{+}, \quad \omega(\zeta)=i \frac{1+\zeta}{1-\zeta}, \quad \zeta \in \mathbb{D} .
$$

Recall (see e.g. [7, Cor. VI.1.3]) that $f \in \operatorname{BMO}(\mathbb{R})$ if and only if $h=f \circ \omega \in$ $\operatorname{BMO}(\mathbb{T})$. Let $h_{n}$ be the Fejer sum (A.4) of $h$, and let $f_{n}=h_{n} \circ \omega^{-1}$. By construction, $f_{n}$ is a rational function; let us prove that $f_{n} \rightarrow f *$-weakly in $\operatorname{BMO}(\mathbb{R})$. For $u \in H^{1}\left(\mathbb{C}_{+}\right)$, let $v \in H^{1}(\mathbb{D})$ be given by

$$
v(\zeta)=-4 \pi(1-\zeta)^{-2} u(\omega(\zeta)) .
$$

Then a direct calculation shows that

$$
T_{f}(u)=t_{h}(\zeta v), \text { and } T_{f_{n}}(u)=t_{h_{n}}(\zeta v) .
$$

Now we get $T_{f_{n}}(u) \rightarrow T_{f}(u)$ by the first step of the proof. Similarly, one proves $T_{\bar{f}_{n}}(u) \rightarrow T_{\bar{f}}(u)$

\section{REFERENCES}

[1] A. B. Aleksandrov, V. V. Peller, Operator Lipschitz functions, Russian Math. Surveys 71 (2016), no. 4, 605-702.

[2] M. Sh. Birman, M. Z. Solomyak, Double Stieltjes operator integrals. (Russian) Problems of mathematical physics. No. 1. Spectral theory and wave processes. (Russian) pp. 33-67. Izdat. Leningrad. Univ., Leningrad, 1966.

[3] M. Sh. Birman, M. Z. Solomyak, Double Operator Integrals in a Hilbert Space, Integr. Equ. Oper. Theory 47 (2003), 131-168.

[4] Yu. L. Daletskit and S. G. Krein, Integration and differentiation of functions of Hermitian operators and applications to the theory of perturbations, (Russian) Voronezh. Gos. Univ. Trudy Sem. Funkcional. Anal. (1956), 1, 81-105. 
[5] R. Frank, A. Pushnitski, Trace class conditions for functions of Schrödinger operators, Comm. Math. Phys. 335 (2015) 477-496.

[6] R. Frank, A. Pushnitski, Schatten class conditions for functions of Schrödinger operators, in preparation.

[7] J. B. Garnett, Bounded analytic functions, Springer, 2007.

[8] I. C. GohberG, M. G. KREǏn, Introduction to the theory of linear nonselfadjoint operators, Translations of Mathematical Monographs, Vol. 18, American Mathematical Society, Providence, R.I. 1969

[9] T. Hytönen, J. van Neerven, M. Veraar, L. Weis, Analysis in Banach spaces, vol. 1: Martingales and Littlewood-Paley Theory, Springer 2016.

[10] T. KAто, Wave operators and similarity for some non-selfadjoint operators, Math. Ann. 162 (1965/1966), 258-279.

[11] T. Kato, Smooth operators and commutators, Studia Math. 31 (1968), 535-546.

[12] Ch. A. MCCARThy, $C_{p}$, Israel J. Math 5 (1967), 249-271.

[13] V. V. PELler, Hankel operators in the theory of perturbations of unitary and self-adjoint operators, Funct. Anal. Appl. 19 (1985) 111-123.

[14] V. Peller, Hankel operators and their applications, Springer, 2003.

[15] D. Potapov, F. Sukochev, Operator-Lipschitz functions in Schatten-von Neumann classes, Acta Math. 207 (2011), no. 2, 375-389.

[16] A. Pushnitski, D. YAfaev, Best rational approximation of functions with logarithmic singularities, Constr. Approx. 2016.

[17] M. Reed, B. Simon, Methods of Modern Mathematical Physics. I Functional Analysis, Revised and enlarged edition, Academic Press, 1980.

[18] R. Rochberg, Toeplitz and Hankel operators on the Paley-Wiener space, Integral Equations and Operator Theory 10 (1987) 187-235.

[19] S. Ju. RotFeld, On singular values of the sum of completely continuous operators, (Russian). Problems of mathematical physics, No. 3: Spectral theory (Russian), pp. 81-87. Izdat. Leningrad. Univ., Leningrad, 1968.

[20] D.YAFAEV, Mathematical Scattering Theory: General Theory, AMS 1992.

(Rupert L. Frank) Mathematisches Institut, Ludwig-Maximilans Universität München, Theresienstr. 39, 80333 München, Germany, and Department of Mathematics, California Institute of Technology, Pasadena, CA 91125, USA

E-mail address: rlfrank@caltech.edu

(Alexander Pushnitski) Department of Mathematics, King's College London, STRAND, London, WC2R 2LS, UK

E-mail address: alexander.pushnitski@kcl.ac.uk 